\title{
Possible signals of vacuum dynamics in the Universe
}

\author{
Joan Solà Peracaula, ${ }^{\star}$ Javier de Cruz Pérez ${ }^{\star}$ and Adrià Gómez-Valent` \\ Departament de Física Quàntica i Astrofísica, and Institute of Cosmos Sciences, Universitat de Barcelona, Av. Diagonal 647, E-08028 Barcelona, \\ Catalonia, Spain
}

Accepted 2018 May 7. Received 2018 May 1; in original form 2018 March 3

\begin{abstract}
We study a generic class of time-evolving vacuum models which can provide a better phenomenological account of the overall cosmological observations as compared to the $\Lambda$ cold dark matter $(\Lambda \mathrm{CDM})$. Among these models, the running vacuum model $(\mathrm{RVM})$ appears to be the most motivated and favoured one, at a confidence level of $\sim 3 \sigma$. We further support these results by computing the Akaike and Bayesian information criteria. Our analysis also shows that we can extract fair signals of dynamical dark energy (DDE) by confronting the same set of data to the generic XCDM and CPL parametrizations. In all cases we confirm that the combined triad of modern observations on baryonic acoustic oscillations, large-scale structure formation, and the cosmic microwave background, provide the bulk of the signal sustaining a possible vacuum dynamics. In the absence of any of these three crucial data sources, the DDE signal cannot be perceived at a significant confidence level. Its possible existence could be a cure for some of the tensions existing in the $\Lambda \mathrm{CDM}$ when confronted to observations.
\end{abstract}

Key words: cosmological parameters-dark energy-large-scale structure of Universecosmology: theory.

\section{INTRODUCTION}

Observations over the years seem to firmly support the current acceleration of the Universe and therefore the possible existence of a generic cause responsible for it which we call dark energy (DE; see e.g. Riess et al. 1998; Perlmutter et al. 1999; WMAP collaboration 2013; Planck collaboration XVI 2014; Planck collaboration XIII 2016; Planck collaboration XIV 2016, and references therein). Cosmologists have worked hard to decipher the dark energy code, but we still ignore the physical nature of the DE and hence of the ultimate cause of the observed acceleration of the Universe. Such theoretical conundrum is the so-called Cosmological Constant Problem (CCP) (Weinberg 1989; Sahni \& Starobinsky 2000; Padmanabhan 2003; Peebles \& Ratra 2003; Copeland, Sami \& Tsujikawa 2006; Solà 2013). In fact, the cosmological constant (CC), $\Lambda$, or equivalently the vacuum energy density associated to it, $\rho_{\Lambda}=\Lambda /(8 \pi G)$ ( $G$ being Newton's gravitational coupling), is usually regarded as the simplest possible explanation for the DE. Historically, the CC was introduced by Einstein in the gravitational field equations $101 \mathrm{yr}$ ago (Einstein 1917). A positive, constant, tiny value (in particle physics units) of order $\rho_{\Lambda} \sim 2.7 \times 10^{-47} \mathrm{GeV}^{4} \sim$ $\left(2.3 \times 10^{-3} \mathrm{eV}\right)^{4}$ can explain the needed speed up of our cosmos according to the observations. The standard or 'concordance' cosmological model embodies such an assumption as a fundamental

\footnotetext{
*E-mail: $\quad$ sola@ fqa.ub.edu(JSP); $\quad$ decruz@fqa.ub.edu(JdCP);
}

built-in principle, together with the hypothesis of dark matter (DM), and for this reason is called the $\Lambda \mathrm{CDM}$ model. Formulated in terms of the current cosmological parameters, the $\Lambda \mathrm{CDM}$ assumes that $\rho_{\Lambda}=$ const. throughout the history of the Universe, with $\Omega_{\Lambda} \simeq 0.7$ and $\Omega_{\mathrm{m}} \simeq 0.3$ at present. Unfortunately, no convincing theoretical explanation is provided about the measured value of $\rho_{\Lambda}$. At the end of the day, no fundamental theory, not even quantum field theory (QFT), can explain this value; and, what is worse, the typical prediction is preposterously large as compared to the measured value. The difficulties inherent to this concept were recognized as of the time when Y.B. Zeldovich first observed (Zeldovich 1967) that the contribution from QFT to the vacuum energy density should be of the order of $\sim m^{4}$ for any quantum field of mass $m$, and therefore many orders of magnitude bigger than the existing upper bound on $\rho_{\Lambda}$ in those days.

Since long cosmologists have felt motivated to look for alternative explanations for the DE beyond a rigid cosmological constant $\Lambda$. The scalar field paradigm was then profusely used also to make the cosmic vacuum dynamical: $\Lambda=\Lambda(\phi)$. In the old days, the main aim was to adjust the large value of $\Lambda$ typically predicted in QFT to be zero. There were many early proposals (see e.g. Endo \& Fukui 1977, 1982; Fujii 1982; Dolgov 1983; Abbott 1985; Zee 1985; Barr 1987; Ford 1987; Peccei, Solà \& Wetterich 1987; Weiss 1987; Barr \& Hochberg 1988). In spite of the hopes raised by these works at solving the 'old CC Problem', it was later shown in Weinberg (1989) through the so-called no-go theorem that most if not all the dynamical adjustment mechanisms existing in the literature to date were plagued by more or less obvious forms of 
subtly hidden fine tuning. For this reason, the subsequent use of scalar fields in cosmology was mostly focused on trying to explain another aspect of the CCP: the cosmic coincidence problem (viz. the fact that $\rho_{\Lambda}$ happens to be so close to the matter density $\rho_{\mathrm{m}}$ right now; see e.g. Peebles \& Ratra 2003). The new wave of dynamical scalar fields in cosmology crystalized in the notions of quintessence, phantom fields and the like, which have had a tremendous influence in cosmology till our days (see e.g. Peebles \& Ratra 1988; Ratra \& Peebles 1988; Wetterich 1988; Wetterich 1995; Caldwell, Dave \& Steinhardt 1998; Zlatev, Wang \& Steinhardt 1999; Amendola 2000; Caldwell, Kamionkowski \& Weinberg 2003), the reviews (Sahni \& Starobinsky 2000; Padmanabhan 2003; Peebles \& Ratra 2003; Copeland, Sami \& Tsujikawa 2006), and the many references therein. At the same time a blooming crest of models based on ascribing a direct phenomenological time-dependence to the CC term, $\Lambda=\Lambda(t)$, broke with impetus into the market. For an account of some of the old attempts, see Overduin \& Cooperstock (1998, and references therein).

In this work, rather than attempting to solve the underlying theoretical enigmas affecting the $\Lambda \mathrm{CDM}$ we wish to address more practical matters. We wish to follow the original phenomenological approach that made possible to unveil that $\rho_{\Lambda}$ is non-vanishing, irrespective of its ultimate physical nature. The method was largely empirical, namely $\rho_{\Lambda}$ was assumed to be a parameter and then fitted directly to the data. Of course a minimal set of assumptions had to be made, such as the validity of the Cosmological Principle and hence of the Friedmann-Lemaître-Robertson-Walker (FLRW) metric, with the ensuing set of Friedmann equations for the scale factor (Peebles 1993). In our case, we wish of course to keep these minimal assumptions and make a phenomenological case study of the possibility that $\Lambda$ might be not just a parameter but a slowly varying cosmic variable mimicking the $\Lambda$ CDM-like behaviour. Furthermore, we motivate our study by considering the possibility that the inherent dynamics in $\rho_{\Lambda}$ is connected to fundamental aspects of QFT. In fact, within the class of dynamical vacuum models (DVMs), one of the main models under study is the 'running vacuum model' (RVM), which can be connected to important aspects of QFT in curved space-time (see Solà 2013; Solà \& Gómez-Valent 2015; Solà 2016; Solà 2008, and references therein). One can think of this framework as one in which the $\Lambda \mathrm{CDM}$ is replaced by $\bar{\Lambda} \mathrm{CDM}$ (Solà \& Gómez-Valent 2015), with $\bar{\Lambda}=\Lambda(H)$, or equivalently $\rho_{\Lambda}=\rho_{\Lambda}(H)$, playing the role of 'running' vacuum energy density. Interestingly, such a running with the expansion rate, $H$, can be related to the renormalization group. For previous investigations along these lines, see e.g. España-Bonet et al. (2003, 2004), Babić et al. (2005), Basilakos, Plionis \& Solà (2009), Solà (2011), Grande et al. (2011), Basilakos, Polarski \& Solà (2012), Basilakos \& Solà (2014), Gómez-Valent, Solà \& Basilakos (2015a), Gómez-Valent \& Solà (2015), Solà, Gómez-Valent \& de Cruz Pérez (2015), GómezValent, Karimkhani \& Solà (2015b), Basilakos (2015), Geng, Lee \& Zhang (2016), and Geng, Lee \& Yin (2017), and the closely related recent works (Solà, Gómez-Valent \& de Cruz Pérez 2017a,b,c,d; Solà, de Cruz Pérez \& Gómez-Valent 2018). It turns out that the peak confidence level for DDE that we find in the context of the DVMs is near $\gtrsim 3.5 \sigma$ at present. Interestingly, when we confront the same data with a simple XCDM (Turner \& White 1997) or CPL (Chevallier \& Polarski 2001; Linder 2003, 2004) parametrizations of the DDE (Amendola \& Tsujikawa 2015) we can still extract significant evidence of vacuum dynamics, showing that the signal is not restricted to particular models but it is rather generic. The first relatively recent signs of such dynamics were advanced in Gómez-Valent et al. (2015a,b), Gómez-Valent \& Solà (2015), and Solà, Gómez-Valent \& de Cruz Pérez (2015). Since then new support to the dynamical DE from the observational point of view has appeared in the literature using different methods and attaining a similar confidence level (Zhao et al. 2017a).

Finally, in view of the practical nature of this work we keep an eye to the fact that the $\Lambda \mathrm{CDM}$ is afflicted of several persistent tensions when compared to the cosmological data. Such tensions involve relevant parameters of cosmology, such as the Hubble parameter, i.e. the current value of the Hubble function, $H\left(t_{0}\right) \equiv H_{0}$, and the current value of the rms of mass fluctuations at spheres of $8 h^{-1} \mathrm{Mpc}$, i.e. $\sigma_{8}(0)$. Such situation could be caused by as-yet unrecognized uncertainties or hint at physics beyond the $\Lambda \mathrm{CDM}$ (Freedman 2017). We cannot exclude e.g. that the peculiarities of important cosmological processes, for instance those related with structure formation, are conspicuously sensitive and even positively receptive to a mild dynamical variation of the cosmic vacuum, which certainly influences the gravitational interaction of matter. Recall that a positive $\Lambda$ suppresses the growth of structure formation and this explains why the $\Lambda \mathrm{CDM}$ model is highly preferred to the CDM one, in which $\Lambda=0$. Therefore, it is natural to reconsider these processes by considering the effect of a time modulation of the growth suppression through $\rho_{\Lambda}=\rho_{\Lambda}(t)$. We find that this helps to ameliorate the description of the large-scale structure (LSS) formation data, so it is worthwhile testing it. For some studies addressing the existing tensions from various perspectives, see e.g. Valentino et al. (2017a), Valentino, Melchiorri \& Mena (2017b), Zhai et al. (2017), Solà, Gómez-Valent \& de Cruz Pérez (2017c,d), and Gómez-Valent \& Solà $(2017,2018)$. See also Chen et al. (2016) and Yu, Ratra \& Wang (2018).

The guidelines of our work are as follows. In Section 2, we describe the DVMs. In Section 3, we report on the set of cosmological data used, on distant Type Ia supernovae (SNIa), baryonic acoustic oscillations (BAOs), the Hubble parameter values at different redshifts, the LSS data, and the cosmic microwave background (CMB) from Planck. In Section 4, we discuss aspects of structure formation with dynamical vacuum. The numerical analysis of the DVMs and a comparison with the standard XCDM and CPL parametrizations is the object of Section 5. An ample discussion of the results along with a reanalysis under different conditions is developed in Section 6. Finally, in Section 7 we present our conclusions.

\section{DYNAMICAL VACUUM MODELS}

The gravitational field equations are $G_{\mu \nu}=8 \pi G \tilde{T}_{\mu \nu}$, where $G_{\mu \nu}=$ $R_{\mu \nu}-\frac{1}{2} g_{\mu \nu} R$ is the Einstein tensor and $\tilde{T}_{\mu \nu} \equiv T_{\mu \nu}+g_{\mu \nu} \rho_{\Lambda}$ is the full energy-momentum tensor involving the effect of both matter and vacuum energy density, with $\rho_{\Lambda}=\Lambda /(8 \pi G)$. The structure of $\tilde{T}_{\mu v}$ shows that the vacuum is dealt with as a perfect fluid carrying an equation of state $(\mathrm{EoS}) p_{\Lambda}=-\rho_{\Lambda}$. When the matter can also be treated as an ideal fluid and is distributed homogeneously and isotropically, as postulated by the Cosmological Principle, we can write $\tilde{T}_{\mu \nu}=\left(\rho_{\Lambda}-p_{m}\right) g_{\mu \nu}+\left(\rho_{m}+p_{m}\right) U_{\mu} U_{\nu}$, where $U_{\mu}$ is the bulk four-velocity of the cosmic fluid, $\rho_{\mathrm{m}}$ is the proper energy density of matter and $p_{\mathrm{m}}$ its isotropic pressure. We assume the standard cosmological framework grounded on the FLRW metric with flat three-dimensional slices: $\mathrm{d} s^{2}=\mathrm{d} t^{2}-a^{2}(t) \mathrm{d} x^{2}$, where $t$ is the cosmic time and $a(t)$ is the scale factor. However, we admit that matter can be in interaction with vacuum, which is tantamount to saying that $\rho_{\Lambda}=\rho_{\Lambda}(\zeta)$ is a function of some cosmic variable evolving with time, $\zeta=\zeta(t)$. While this, of course, implies that $\dot{\rho}_{\Lambda} \equiv \mathrm{d} \rho_{\Lambda} / \mathrm{d} t \neq 0$, we assume that $\dot{G}=0$ in our study (see Solà, Gómez-Valent \& de Cruz Pérez 2015, 2017a, for studies including the option $\dot{G} \neq 0$ ). 
Such vacuum dynamics is compatible with the Bianchi identity (see below) provided there is some energy exchange between vacuum and matter. It means that matter cannot be strictly conserved in these circumstances. The standard Friedmann and acceleration equations for the present Universe remain formally identical to the standard $\Lambda \mathrm{CDM}$ case:

$$
\begin{aligned}
& 3 H^{2}=8 \pi G \sum_{N} \rho_{N}=8 \pi G\left(\rho_{m}+\rho_{r}+\rho_{\Lambda}(\zeta)\right) \\
& 3 H^{2}+2 \dot{H}=-8 \pi G \sum_{N} p_{N}=-8 \pi G\left(p_{r}-\rho_{\Lambda}(\zeta)\right) .
\end{aligned}
$$

Here, $H=\dot{a} / a$ is the usual Hubble function, $\rho_{\mathrm{m}}=\rho_{\mathrm{b}}+\rho_{\mathrm{dm}}$ involves the pressureless contributions from baryons and cold DM, and $\rho_{\mathrm{r}}$ is the radiation density (with the usual EoS $p_{r}=\rho_{\mathrm{r}} / 3$ ). We emphasize once more that in the above equations we stick to the EoS $p_{\Lambda}=-\rho_{\Lambda}$, although the vacuum is dynamical, $\rho_{\Lambda}(t)=\rho_{\Lambda}(\zeta(t))$, and its evolution is tied to the cosmic expansion. The sums above run over all the components $N=d m, b, r, \Lambda$. In all of the DVMs being considered here, the cosmic variable $\zeta$ is either the scale factor or can be expressed analytically in terms of it, $\zeta=\zeta(a)$, or equivalently in terms of the cosmological redshift, $z=a^{-1}-1$, in which we adopt the normalization $a=1$ at present. From the basic pair of equations (1) and (2), a first integral of the system follows:

$$
\begin{aligned}
& \sum_{N} \dot{\rho}_{N}+3 H\left(\rho_{N}+p_{N}\right) \\
& =\dot{\rho}_{\Lambda}+\dot{\rho}_{d m}+3 H \rho_{d m}+\dot{\rho}_{b}+3 H \rho_{b}+\dot{\rho}_{r}+4 H \rho_{r}=0 .
\end{aligned}
$$

Such a first integral ensues also from the divergenceless property of the full energy-momentum tensor $\tilde{T}_{\mu \nu}$ in the FLRW metric, i.e. $\nabla^{\mu} \tilde{T}_{\mu \nu}=0$. The last property is a consequence of the Bianchi identity satisfied by the Einstein tensor, $\nabla^{\mu} G_{\mu \nu}=0$, and the assumed constancy of the Newtonian coupling $G$. It reflects the local conservation law of the compound system formed by matter and vacuum, and the consequent non-conservation of each of these components when taken separately.

The concordance model assumes that matter and radiation are self-conserved after equality. It also assumes that baryons and CDM are separately conserved. Hence their respective energy densities satisfy $\dot{\rho}_{b}+3 H \rho_{b}=0, \dot{\rho}_{r}+4 H \rho_{r}=0$ and $\dot{\rho}_{d m}+3 H \rho_{d m}=0$. In the presence of vacuum dynamics, it is obvious that at least one of these equations cannot hold. Following our definite purpose to remain as close as possible to the $\Lambda \mathrm{CDM}$, we shall assume that the first two of the mentioned conservation equations still hold good but that the last does not, meaning that the vacuum exchanges energy only with DM. The dilution laws for baryons and radiation as a function of the scale factor therefore take on the conventional $\Lambda \mathrm{CDM}$ forms:

$\rho_{b}(a)=\rho_{b 0} a^{-3}, \quad \rho_{r}(a)=\rho_{r 0} a^{-4}$,

where $\rho_{b 0}$ and $\rho_{r 0}$ are the corresponding current values. In contrast, the density of DM is tied to the dynamics of the vacuum. Taking into account the conserved components and introducing the vacuumdark matter interaction source, $Q$, we can write the interactive part of (3) into two coupled equations:

$\dot{\rho}_{d m}+3 H \rho_{d m}=Q, \quad \dot{\rho}_{\Lambda}=-Q$

The solution of these equations will depend on the particular form assumed for $Q$, which determines the leakage rate of vacuum energy into dark matter or vice versa. Such a leakage must certainly be much smaller than the standard dilution rate of non-relativistic matter associated to the cosmic expansion (i.e. much smaller than $\sim a^{-3}$ ), as otherwise these anomalous effects would be too sharp at the present time. Therefore, we must have $0<|Q| \ll \dot{\rho}_{m}$. The different DVMs will be characterized by different functions $Q_{i}(i=1,2, \ldots)$.

Two possible phenomenological ansatzes considered in the literature are (Salvatelli et al. 2014; Murgia, Gariazzo \& Fornengo 2016; Li, Zhang \& Zhang 2016; Zhao et al. 2017; Guo, Zhang \& Zhang 2018)

Model $Q_{d m}: \quad Q_{d m}=3 v_{d m} H \rho_{d m}$

Model $Q_{\Lambda}: \quad Q_{\Lambda}=3 v_{\Lambda} H \rho_{\Lambda}$.

The dimensionless parameters $v_{i}=\left(v_{d m}, v_{\Lambda}\right)$ for each model $\left(Q_{d m}\right.$, $Q_{\Lambda}$ ) determine the strength of the dark-sector interaction in the sources $Q_{i}$ and enable the evolution of the vacuum energy density. For $v_{i}>0$, the vacuum decays into DM (which is thermodynamically favourable Salim \& Waga 1993; Lima 1996) whereas for $v_{i}<0$ is the other way around. This is also a relevant argument to judge the viability of these models, as only the first situation is compatible with the second law of thermodynamics. There are many more choices for $Q$ (see e.g. Bolotin et al. 2015; Costa et al. 2017), but it will suffice to focus on these models and the RVM one defined in the next section to effectively assess the possible impact of the DVMs in the light of the modern observational data.

\subsection{The running vacuum model (RVM)}

The last DVM under study is the so-called running vacuum model (RVM), which can be motivated in the context of QFT in curved space-time (cf. Solà 2013; Solà \& Gómez-Valent 2015, and references therein). The model has some virtues and can be extended to afford an effective description of the cosmic evolution starting from inflation up to our days (Lima, Basilakos \& Solà 2013, 2015, 2016; Perico et al. 2013; Solà 2013, 2015; Solà \& Gómez-Valent 2015). For instance, in Solà (2015) it is suggested that the RVM could positively impinge on solving some of the fundamental cosmological problems, including the entropy problem. Intriguingly, the inherent tiny leakage of vacuum energy into matter within the RVM could also furnish an explanation for the possible slow time variation of the fundamental constants, an issue that has been examined in detail in Fritzsch \& Solà $(2012,2015)$ and Fritzsch, Solà \& Nunes (2017). See also the old work (Terazawa 1981). We shall not discuss here the implications for the early Universe, but only for the part of the cosmic history that is accessible to our measurements and can therefore be tested phenomenologically with the current data.

As advertised, for the specific RVM case the cosmic variable $\zeta$ in the field equations (1) and (2) can be identified with the Hubble rate $H$. The form of the RVM for the post-inflationary epoch and hence relevant for the current Universe reads as follows:

$\rho_{\Lambda}(H)=\frac{3}{8 \pi G}\left(c_{0}+v H^{2}\right)$.

Such structure can be linked to a renormalization group (RG) equation for the vacuum energy density, in which the running scale $\mu$ of the RG is associated with the characteristic energy scale of the FLRW metric, i.e. $\mu=H$. The additive constant $c_{0}=H_{0}^{2}\left(\Omega_{\Lambda}-v\right)$ appears because one integrates the RG equation satisfied by $\rho_{\Lambda}(H)$. It is fixed by the boundary condition $\rho_{\Lambda}\left(H_{0}\right)=\rho_{\Lambda 0}$, where $\rho_{\Lambda 0}$ and $H_{0}$ are the current values of these quantities; similarly $\Omega_{\Lambda}=\rho_{\Lambda 0} / \rho_{c 0}$ and $\rho_{c 0}=3 H_{0}^{2} /(8 \pi G)$ are the values of the vacuum density parameter and the critical density today. The dimensionless coefficient $v$ encodes the dynamics of the vacuum at low energy and 
can be related with the $\beta$-function of the running of $\rho_{\Lambda}$. Thus, we naturally expect $|v| \ll 1$. An estimate of $v$ at one loop in QFT indicates that is of the order of $10^{-3}$ at most (Solà 2008), but here we will treat it as a free parameter. This means we shall deal with the RVM on pure phenomenological grounds, hence fitting actually $v$ to the observational data (cf. Section 3).

In the RVM case, the source function $Q$ in (5) is not just put by hand (as in the case of the DVMs introduced before). It is a calculable expression from (8), using Friedmann's equation (1) and the local conservation laws (4)-(5). We find

RVM: $Q=-\dot{\rho}_{\Lambda}=v H\left(3 \rho_{m}+4 \rho_{r}\right)$,

where we recall that $\rho_{m}=\rho_{d m}+\rho_{b}$, and that $\rho_{b}$ and $\rho_{r}$ are known functions of the scale factor - see equation (4). The remaining densities, $\rho_{d m}$ and $\rho_{\Lambda}$, must be determined upon further solving the model explicitly, see Section 2.2. If baryons and radiation would also possess a small interaction with vacuum and/or $G$ would evolve with time, the cosmological solutions would be different (Basilakos 2015; Gómez-Valent \& Solà 2015; Gómez-Valent et al. 2015a; Solà, Gómez-Valent \& de Cruz Pérez 2015, 2017a). We can see from (9) that the parameter $v$ plays a similar role as $\left(v_{d m}, v_{\Lambda}\right)$ for the more phenomenological models (6) and (7). The three of them will be collectively denoted $v_{i}$.

\subsection{Solving explicitly the dynamical vacuum models}

The matter and vacuum energy densities of the DVMs can be computed straightforwardly upon solving the coupled system of differential equations (5), given the previous explicit forms for the interacting source in each case and keeping in mind that, in the current framework, the baryon $\left(\rho_{b}\right)$ and radiation $\left(\rho_{r}\right)$ parts are separately conserved. After some calculations the equations for the DM energy densities $\rho_{d m}$ for each model (RVM, $Q_{d m}, Q_{\Lambda}$ ) can be solved in terms of the scale factor. Below we quote the final results for each case:

$\mathrm{RVM}: \quad \rho_{d m}(a)=\rho_{d m 0} a^{-3(1-v)}+\rho_{b 0}\left(a^{-3(1-v)}-a^{-3}\right)$

$$
+\frac{4 v}{1+3 v} \rho_{r 0}\left(a^{-3(1-v)}-a^{-4}\right)
$$

$Q_{\mathrm{dm}}: \quad \rho_{d m}(a)=\rho_{d m 0} a^{-3\left(1-v_{d m}\right)}$

$\mathrm{Q}_{\Lambda}: \quad \rho_{d m}(a)=\rho_{d m 0} a^{-3}+\frac{v_{\Lambda}}{1-v_{\Lambda}} \rho_{\Lambda 0}\left(a^{-3 v_{\Lambda}}-a^{-3}\right)$

In solving the differential equations (5) we have traded the cosmic time variable for the scale factor using the chain rule $\mathrm{d} / \mathrm{d} t=a H \mathrm{~d} / \mathrm{d} a$. The corresponding vacuum energy densities can also be solved in the same variable, and yield

$\begin{aligned} \mathrm{RVM}: \quad \rho_{\Lambda}(a)= & \rho_{\Lambda 0}+\frac{v \rho_{m 0}}{1-v}\left(a^{-3(1-v)}-1\right) \\ & +\frac{v \rho_{r} 0}{1-v}\left(\frac{1-v}{1+3 v} a^{-4}+\frac{4 v}{1+3 v} a^{-3(1-v)}-1\right)\end{aligned}$

$Q_{d m}: \quad \rho_{\Lambda}(a)=\rho_{\Lambda 0}+\frac{v_{d m} \rho_{d m 0}}{1-v_{d m}}\left(a^{-3\left(1-v_{d m}\right)}-1\right)$

$Q_{\Lambda}: \quad \rho_{\Lambda}(a)=\rho_{\Lambda 0} a^{-3 v_{\Lambda}}$

One can easily check that for $a=1$ (i.e. at the present epoch) all of the energy densities (10)-(15) recover their respective current values $\rho_{N 0}(N=d m, \Lambda)$. In addition, for $v_{i} \rightarrow 0$ we retrieve for the three DM densities the usual $\Lambda \mathrm{CDM}$ expression $\rho_{d m}(a)=\rho_{d m 0} a^{-3}$, and the corresponding vacuum energy densities $\rho_{\Lambda}(a)$ boil down to the constant value $\rho_{\Lambda 0}$ in that limit. The normalized Hubble rate $E \equiv$ $H / H_{0}$ for each model can be easily obtained by plugging the above formulas, along with the radiation and baryon energy densities (4), into Friedmann's equation (1). We find

$$
\begin{aligned}
\mathrm{RVM}: \quad E^{2}(a)=1 & +\frac{\Omega_{m}}{1-v}\left(a^{-3(1-v)}-1\right) \\
& +\frac{\Omega_{r}}{1-v}\left(\frac{1-v}{1+3 v} a^{-4}+\frac{4 v}{1+3 v} a^{-3(1-v)}-1\right)
\end{aligned}
$$

$$
\begin{aligned}
Q_{d m}: \quad E^{2}(a)= & 1+\Omega_{b}\left(a^{-3}-1\right) \\
& +\frac{\Omega_{d m}}{1-v_{d m}}\left(a^{-3\left(1-v_{d m}\right)}-1\right)+\Omega_{r}\left(a^{-4}-1\right)
\end{aligned}
$$

$$
\begin{aligned}
Q_{\Lambda}: \quad E^{2}(a)= & \frac{a^{-3 v_{\Lambda}}-v_{\Lambda} a^{-3}}{1-v_{\Lambda}}+\frac{\Omega_{m}}{1-v_{\Lambda}}\left(a^{-3}-a^{-3 v_{\Lambda}}\right) \\
& +\Omega_{r}\left(a^{-4}+\frac{v_{\Lambda}}{1-v_{\Lambda}} a^{-3}-\frac{a^{-3 v_{\Lambda}}}{1-v_{\Lambda}}\right)
\end{aligned}
$$

In the above expressions, we have used the cosmological parameters $\Omega_{N}=\rho_{N 0} / \rho_{c 0}$ for each fluid component $(N=\mathrm{dm}, b, r, \Lambda)$, and defined $\Omega_{\mathrm{m}}=\Omega_{d m}+\Omega_{\mathrm{b}}$. Altogether, they satisfy the sum rule $\sum_{N} \Omega_{N}=1$. The normalization condition $E(1)=1$ in these formulas is apparent, meaning that the Hubble function for each model reduces to $H_{0}$ at present, as they should; and, of course, for $v_{i} \rightarrow 0$ we recover the $\Lambda \mathrm{CDM}$ form for $H$, as should be expected.

From the structure of equations (13) and (16), we can readily see that the vacuum energy density for the RVM can be fully written as a function of a cosmic variable $\zeta$, which can be chosen to be not only the scale factor but the full Hubble function $\zeta=H$. The result is, of course, equation (8). In contrast, for the $Q_{d m}$ and $Q_{\Lambda}$ models this is not possible, as it is clear on comparing equations (14) and (15) and the corresponding ones (17) and (18). For these models, $\rho_{\Lambda}$ can only be written as a function of the scale factor. Thus, the RVM happens to have the greatest level of symmetry since its origin is an RG equation in $H$ whose solution is precisely (8).

\subsection{XCDM and CPL parametrizations}

Together with the DVMs, we fit also the same data through the simple XCDM parametrization of the dynamical DE, first introduced in Turner \& White (1997). Since both matter and DE are selfconserved (i.e. they are not interacting), the DE density as a function of the scale factor is simply given by $\rho_{X}(a)=\rho_{X 0} a^{-3\left(1+w_{0}\right)}$, with $\rho_{X 0}=\rho_{\Lambda 0}$, where $w_{0}$ is the (constant) EoS parameter of the generic DE entity $\mathrm{X}$ in this parametrization. The normalized Hubble function is

$E^{2}(a)=\Omega_{m} a^{-3}+\Omega_{r} a^{-4}+\Omega_{\Lambda} a^{-3\left(1+w_{0}\right)}$.

For $w_{0}=-1$, it boils down to that of the $\Lambda \mathrm{CDM}$ with rigid $\mathrm{CC}$ term. The use of the XCDM parametrization throughout our analysis will be useful to roughly mimic a (non-interactive) DE scalar field with constant EoS. For $w_{0} \gtrsim-1$, the XCDM mimics quintessence, whereas for $w_{0} \lesssim-1$ it mimics phantom DE. 
Table 1. Best-fitting values for the $\Lambda$ CDM, XCDM, CPL, and the three dynamical vacuum models (DVMs). The specific fitting parameters for each DVM are $v_{i}=v(\mathrm{RVM}), v_{\mathrm{dm}}\left(Q_{\mathrm{dm}}\right)$ and $v_{\Lambda}\left(Q_{\Lambda}\right)$, whilst for the XCDM and CPL are the EoS parameters $w_{0}$ and the pair $\left(w_{0}, w_{1}\right)$, respectively. For the DVMs and the $\Lambda \mathrm{CDM}$, we have $w_{0}=-1$ and $w_{1}=0$. The remaining parameters are as in the $\Lambda \mathrm{CDM}$ and are not shown. For convenience, we reckon the values of $\sigma_{8}(0)$ for each model, which are not part of the fit but are computed from the fitted ones following the procedure indicated in Section 4.3. The (positive) increments $\triangle \mathrm{AIC}$ and $\triangle \mathrm{BIC}$ (see the main text, Section 5.2) clearly favour the DDE options. The RVM and $Q_{\mathrm{dm}}$ are particularly favoured ( $\sim 3.8 \sigma$ c.l. and $3.6 \sigma$, respectively). Our fit is performed over a rich and fully updated SNIa+BAO+H(z)+LSS+CMB data set (cf. Section 3).

\begin{tabular}{|c|c|c|c|c|c|c|c|c|}
\hline Model & $h$ & $\Omega_{\mathrm{m}}$ & $v_{i}$ & $w_{0}$ & $w_{1}$ & $\sigma_{8}(0)$ & $\Delta \mathrm{AIC}$ & $\triangle \mathrm{BIC}$ \\
\hline XCDM & $0.672 \pm 0.007$ & $0.311 \pm 0.007$ & - & $-0.923 \pm 0.023$ & - & $0.767 \pm 0.014$ & 8.55 & 6.31 \\
\hline RVM & $0.677 \pm 0.005$ & $0.303 \pm 0.005$ & $0.00158 \pm 0.00042$ & -1 & - & $0.736 \pm 0.019$ & 12.91 & 10.67 \\
\hline$Q_{\mathrm{dm}}$ & $0.678 \pm 0.005$ & $0.302 \pm 0.005$ & $0.00216 \pm 0.00060$ & -1 & - & $0.740 \pm 0.018$ & 12.13 & 9.89 \\
\hline$Q_{\Lambda}$ & $0.691 \pm 0.004$ & $0.298 \pm 0.005$ & $0.00601 \pm 0.00253$ & -1 & - & $0.790 \pm 0.010$ & 3.41 & 1.17 \\
\hline
\end{tabular}

A slightly more sophisticated approximation to the behaviour of a non-interactive scalar field playing the role of dynamical DE is afforded by the CPL parametrization (Chevallier \& Polarski 2001; Linder 2003, 2004), in which one assumes that the generic DE entity $X$ has a slowly varying EoS of the form

$w_{D}=w_{0}+w_{1}(1-a)=w_{0}+w_{1} \frac{z}{1+z}$.

The CPL parametrization, in contrast to the XCDM one, makes allowance for a time evolution of the dark energy EoS owing to the presence of the additional parameter $w_{1}$, which satisfies $0<\left|w_{1}\right| \ll$ $\left|w_{0}\right|$, with $w_{0} \gtrsim-1$ or $w_{0} \lesssim-1$. The expression (20) is seen to have a well-defined limit both in the early Universe $(a \rightarrow 0$, equivalently $z \rightarrow \infty)$ and in the current one $(a=1$, or $z=0)$. The corresponding normalized Hubble function for the CPL can be easily found:

$E^{2}(a)=\Omega_{m} a^{-3}+\Omega_{r} a^{-4}+\Omega_{\Lambda} a^{-3\left(1+w_{0}+w_{1}\right)} e^{-3 w_{1}(1-a)}$.

The XCDM and the CPL parametrizations can be conceived as a kind of baseline frameworks to be referred to in the study of dynamical DE. We expect that part of the DDE effects departing from the $\Lambda \mathrm{CDM}$ should be captured by these parametrizations, either in the form of effective quintessence behaviour $(w \gtrsim-1)$ or effective phantom behaviour $(w \lesssim-1)$. The XCDM, though, is the most appropriate for a fairer comparison with the DVMs, all of which also have one single vacuum parameter $v_{i}$.

\section{DATA SETS AND RESULTS}

In this work, we fit the $\Lambda$ CDM, XCDM, CPL, and the three DVMs to the cosmological data from Type Ia supernovae (Betoule et al. 2014), BAOs (Beutler et al. 2011; Kazin et al. 2014; Aubourg et al. 2015; Delubac et al. 2015; Ross et al. 2015; Gil-Marín et al. 2017), the values of the Hubble parameter extracted from cosmic chronometers at various redshifts, $H\left(z_{i}\right)$ (Jiménez et al. 2003; Simon, Verde \& Jiménez 2005; Stern et al. 2010; Moresco et al. 2012, 2016; Zhang et al. 2014; Moresco 2015), the CMB data from Planck 2015 (Planck collaboration XIII 2016) and the most updated set of LSS formation data embodied in the quantity $f\left(z_{i}\right) \sigma_{8}\left(z_{i}\right)$; see the corresponding values and references in Table2. It turns out that the LSS data is very important for the DDE signal, and up to some updating performed here it has been previously described in more detail in Solà, Gómez-Valent \& de Cruz Pérez (2017a). We denote this string of cosmological data by SNIa+BAO+H(z)+LSS+CMB.

A guide to the presentation of our results is the following. The various fitting analyses and contour plots under different conditions (to be discussed in detail in the next sections) are displayed in four fitting tables, Tables 1 and 3-5, and in seven figures, Figs 1-7.
Table 2. Published values of $f(z) \sigma_{8}(z)$, referred to in the text as the LSS formation data.

\begin{tabular}{lccc}
\hline Survey & $z$ & $f(z) \sigma_{8}(z)$ & References \\
\hline 6dFGS & 0.067 & $0.423 \pm 0.055$ & Beutler et al. (2012) \\
SDSS-DR7 & 0.10 & $0.37 \pm 0.13$ & Feix, Nusser \& Branchini (2015) \\
GAMA & 0.18 & $0.29 \pm 0.10$ & Simpson et al. (2016) \\
& 0.38 & $0.44 \pm 0.06$ & Blake et al. (2013) \\
DR12 BOSS & 0.32 & $0.427 \pm 0.056$ & Gil-Marín et al. (2017) \\
& 0.57 & $0.426 \pm 0.029$ & \\
WiggleZ & 0.22 & $0.42 \pm 0.07$ & Blake et al. (2011) \\
& 0.41 & $0.45 \pm 0.04$ & \\
& 0.60 & $0.43 \pm 0.04$ & \\
2MTF & 0.78 & $0.38 \pm 0.04$ & Springob et al. (2016) \\
VIPERS & 0.02 & $0.34 \pm 0.04$ & Granett et al. (2015) \\
VVDS & 0.7 & $0.38_{-0.07}^{+0.06}$ & Guzzo et al. (2008); Song \& \\
& 0.77 & $0.49 \pm 0.18$ & Percival (2009) \\
\hline
\end{tabular}

The main numerical results of our analysis are those recorded in Table 1. Let us mention in particular Fig. 6, whose aim is to identify what are the main data responsible for the DDE effect under study. Bearing in mind the aforementioned significance of the LSS data, Fig. 7 is aimed to compare in a graphical way the impact of the $f(z) \sigma_{8}(z)$ and weak lensing data on our results. The remaining tables and figures contain complementary information, which can be helpful for a more detailed picture of our rather comprehensive study. Worth noticing are the results displayed in Table 5, which shows what would be the outcome of our analysis if we would restrict ourselves to the fitting data employed by the Planck 2015 collaboration (Planck collaboration XIII 2016), where e.g. no LSS data were used and no DDE signal was reported. Additional details and considerations are furnished of course in the rest of our exposition.

\section{STRUCTURE FORMATION WITH DYNAMICAL VACUUM}

Despite the theory of cosmological perturbations has been discussed at length in several specialized textbooks (see e.g. Peebles 1993; Liddle \& Lyth 2000, 2009; Dodelson 2003), the dynamical character of the vacuum produces some changes on the standard equations which are worth mentioning. At deep subhorizon scales, one can show that the matter density contrast $\delta_{m}=\delta \rho_{m} / \rho_{m}$ 
Table 3. Same as in Table 1, but removing the LSS data set from our fitting analysis.

\begin{tabular}{lcccrrr}
\hline Model & $h$ & $\Omega_{\mathrm{m}}$ & $v_{i}$ & $w$ & \multicolumn{1}{c}{$\Delta$ AIC } & - \\
\hline$\Lambda \mathrm{CDM}$ & $0.685 \pm 0.004$ & $0.304 \pm 0.005$ & - & -1 & - \\
$\mathrm{XCDM}$ & $0.684 \pm 0.009$ & $0.305 \pm 0.007$ & - & $-0.992 \pm 0.040$ & -2.25 \\
$\mathrm{RVM}$ & $0.684 \pm 0.007$ & $0.304 \pm 0.005$ & $0.00014 \pm 0.00103$ & -1 & -4.29 \\
$Q_{\mathrm{dm}}$ & $0.685 \pm 0.007$ & $0.304 \pm 0.005$ & $0.00019 \pm 0.00126$ & -1 & -2.27 & -2.27 \\
$Q_{\Lambda}$ & $0.686 \pm 0.004$ & $0.304 \pm 0.005$ & $0.00090 \pm 0.00330$ & -1 & -4.31 & -2.21 \\
\hline
\end{tabular}

Table 4. Same as in Table 1 but removing the CMB data set from our fitting analysis.

\begin{tabular}{|c|c|c|c|c|c|c|}
\hline Model & $h$ & $\Omega_{\mathrm{m}}$ & $v_{i}$ & $w$ & $\Delta \mathrm{AIC}$ & $\triangle \mathrm{BIC}$ \\
\hline XCDM & $0.674 \pm 0.007$ & $0.298 \pm 0.009$ & - & $-0.960 \pm 0.038$ & -1.18 & -3.40 \\
\hline$Q_{d m}$ & $0.677 \pm 0.008$ & $0.296 \pm 0.015$ & $0.00086 \pm 0.00228$ & -1 & -2.10 & -4.32 \\
\hline$Q_{\Lambda}$ & $0.679 \pm 0.005$ & $0.297 \pm 0.013$ & $0.00463 \pm 0.00922$ & -1 & -1.98 & -4.20 \\
\hline
\end{tabular}

Table 5. As in Table 1, but using the same data set as the Planck Collaboration (Planck collaboration XIV 2016).

\begin{tabular}{|c|c|c|c|c|c|c|}
\hline Model & $h$ & $\Omega_{m}$ & $v_{i}$ & $w$ & $\Delta \mathrm{AIC}$ & $\triangle \mathrm{BIC}$ \\
\hline $\mathrm{XCDM}$ & $0.684 \pm 0.010$ & $0.299 \pm 0.009$ & - & $-0.961 \pm 0.033$ & -1.20 & -2.39 \\
\hline$Q_{\mathrm{dm}}$ & $0.686 \pm 0.008$ & $0.297 \pm 0.008$ & $0.00108 \pm 0.00088$ & -1 & -1.02 & -2.21 \\
\hline$Q_{\Lambda}$ & $0.694 \pm 0.006$ & $0.293 \pm 0.007$ & $0.00167 \pm 0.00471$ & -1 & -2.45 & -3.64 \\
\hline
\end{tabular}
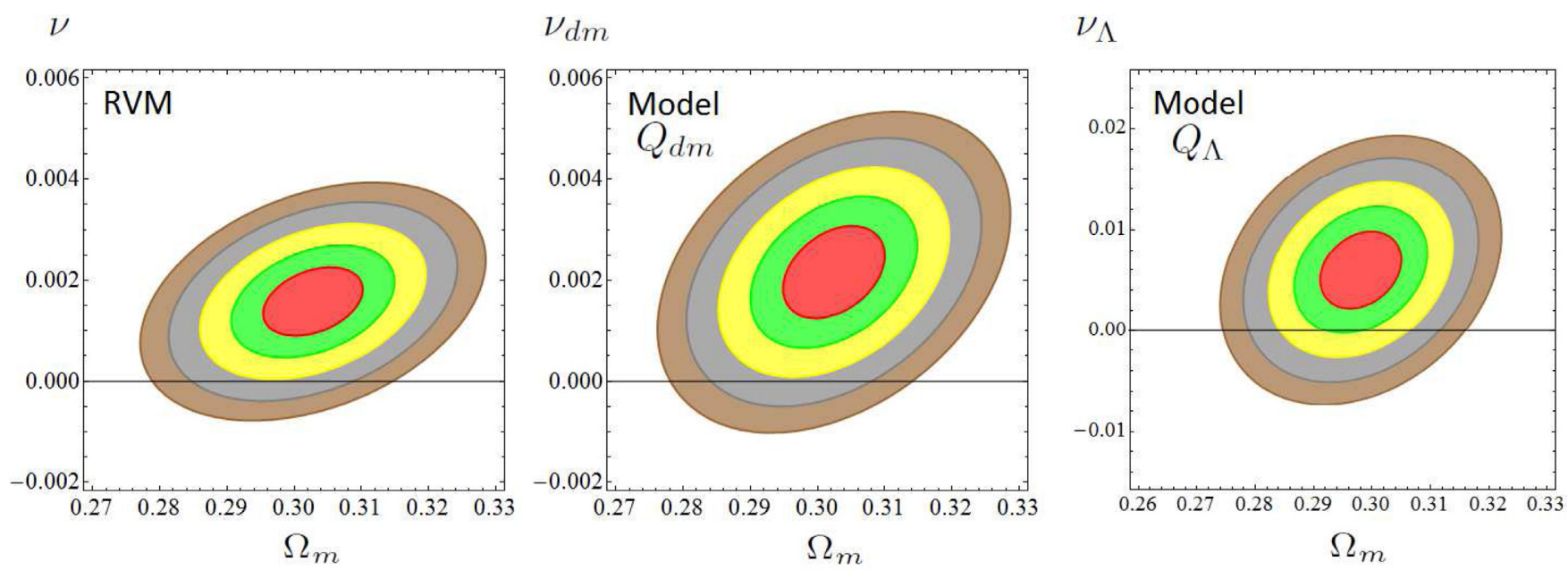

Figure 1. Likelihood contours for the DVMs in the $\left(\Omega_{\mathrm{m}}, v_{i}\right)$ plane for the values $-2 \ln \mathcal{L} / \mathcal{L}_{\max }=2.30,6.18,11.81,19.33,27.65$ (corresponding to $1 \sigma, 2 \sigma$, $3 \sigma, 4 \sigma$, and $5 \sigma$ c.l.) after marginalizing over the rest of the fitting parameters. We estimate that for the RVM, 94.80 per cent (respectively, 89.16 per cent) of the $4 \sigma$ (respectively, $5 \sigma$ ) area is in the $v>0$ region. For the $\mathrm{Q}_{\mathrm{dm}}$ we find that 95.24 per cent (respectively, 89.62 per cent) of the $4 \sigma$ (respectively, $5 \sigma$ ) area is in the $v_{d m}>0$ region. Finally, for the $Q_{\Lambda}$ we estimate that 99.45 per cent (respectively, 90.22 per cent) of the $2 \sigma$ (respectively, $3 \sigma$ ) area is in the $v_{\Lambda}>0$ region. Subsequent marginalization over $\Omega_{\mathrm{m}}$ increases slightly the c.l. and renders the fitting values collected in Table 1 . The $\Lambda$ CDM $\left(v_{i}=0\right)$ appears disfavoured at $\sim 4 \sigma$ c.l. in the RVM and $Q_{\mathrm{dm}}$, and at $\sim 2.5 \sigma$ c.l. for $Q_{\Lambda}$.

obeys the following differential equation (cf. Basilakos \& Solà 2014; Gómez-Valent et al. 2015a; Gómez-Valent \& Solà 2018 for details):

$\ddot{\delta}_{m}+(2 H+\Psi) \dot{\delta}_{m}-\left(4 \pi G \rho_{m}-2 H \Psi-\dot{\Psi}\right) \delta_{m}=0$,

where $\Psi \equiv-\dot{\rho}_{\Lambda} / \rho_{m}=Q / \rho_{m}$, and the (vacuum-matter) interaction source $Q$ for each DVM is given in Section 2. For $\rho_{\Lambda}=$ const. and for the XCDM and CPL there is no such an interaction and equation (22) reduces to $\ddot{\delta}_{m}+2 H \dot{\delta}_{m}-4 \pi G \rho_{m} \delta_{m}=0$, i.e. the $\Lambda \mathrm{CDM}$ form (Peebles 1993). We note that at the scales under consideration we are neglecting the perturbations of the vacuum energy density in front of the perturbations of the matter field. The justification for this has recently been analysed in detail (cf. Gómez-Valent \& Solà 2018).

Let us briefly justify by two alternative methods, the modified form (22), in which the variation of $\rho_{\Lambda}$ enters through the Hubble function and the background quantity $\Psi$, but not through any perturbed quantity. We shall conveniently argue in the context of 


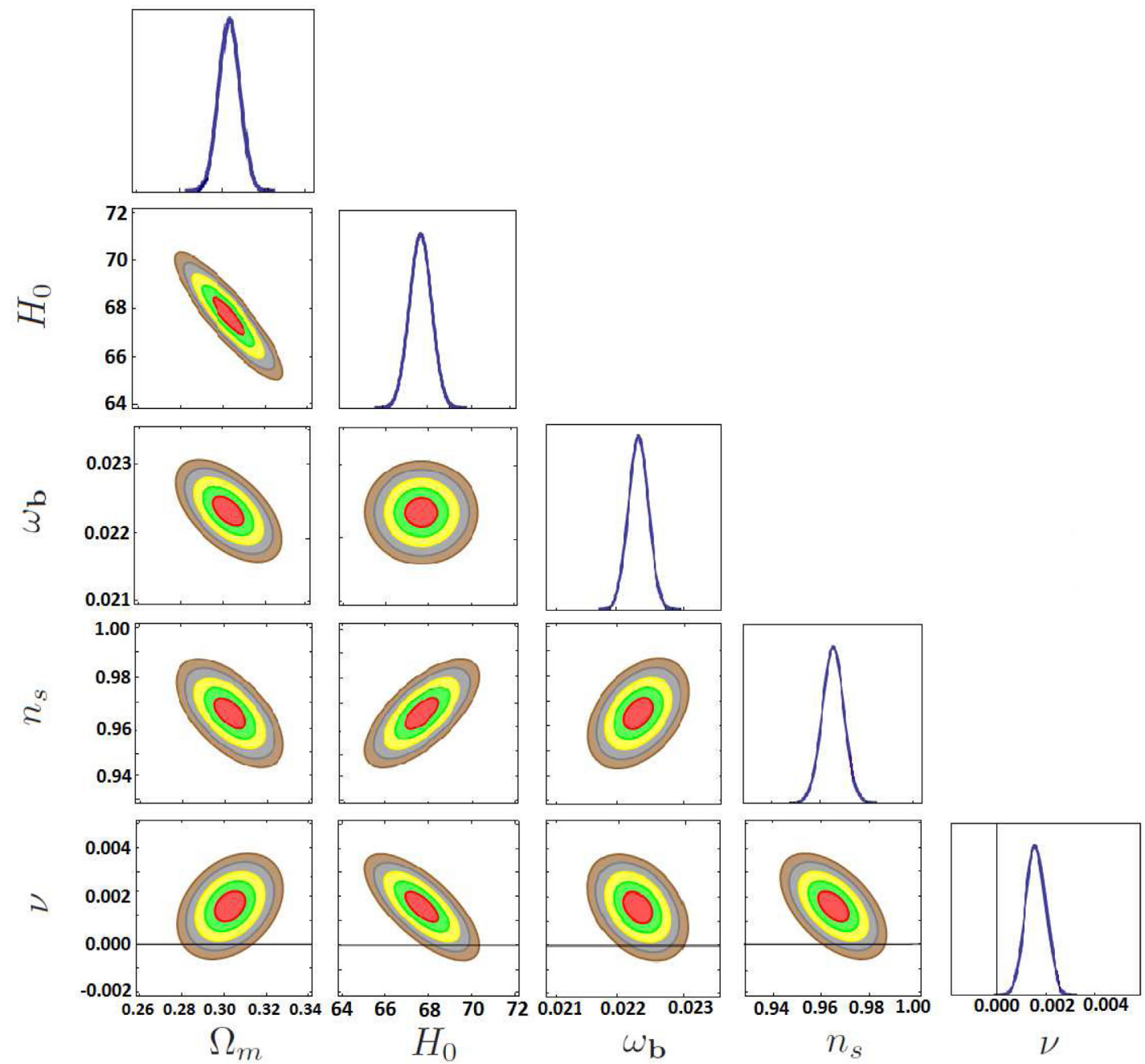

Figure 2. As in Fig. 1, but projecting the fitting results of the RVM onto the different planes of the involved parameters $\left(H_{0}\right.$ is expressed in $\left.\mathrm{Km} \mathrm{s}^{-1} \mathrm{Mpc}^{-1}\right)$. The horizontal line $v=0$ in the plots of the last row corresponds to the $\Lambda$ CDM. It is apparent that it is significantly excluded at $\sim 4 \sigma$ c.l. in all cases. The peak in the rightmost plot corresponds to the central value $\nu=0.00158$ indicated in Table 1.

two well-known gauges, the synchronous gauge and the Newtonian conformal gauge, thus providing a two-fold justification. In the synchronous gauge, vacuum perturbations $\delta \rho_{\Lambda}$ modify the momentum conservation equation for the matter particles in a way that we can easily get e.g. from the general formulae of Gómez-Valent \& Solà (2018) and Grande, Pelinson \& Solà (2009), with the result

$\dot{v}_{m}+H v_{m}=\frac{1}{a} \frac{\delta \rho_{\Lambda}}{\rho_{m}}-\Psi v_{m}$,

where $v=\nabla v_{m}$ is the associated peculiar velocity, with potential $v_{m}$ (note that this quantity has dimension of inverse energy in natural units). By setting $\delta \rho_{\Lambda}=a Q v_{m}=a \rho_{m} \Psi v_{m}$, the two terms on the right-hand side of (23) cancel each other and we recover the corresponding equation of the $\Lambda \mathrm{CDM}$. On the other hand, in the Newtonian or conformal gauge (Mukhanov, Feldman \& Brandenberger 1992; Ma \& Bertschinger 1995) we find a similar situation. The analogue of the previous equation is the modified Euler's equation in the presence of dynamical vacuum energy,

$\frac{\mathrm{d}}{\mathrm{d} \eta}\left(\rho_{m} v_{m}\right)+4 \mathcal{H} \rho_{m} v_{m}+\rho_{m} \phi-\delta \rho_{\Lambda}=0$ where $\phi$ is the gravitational potential that appears explicitly in the Newtonian conformal gauge, and $\eta$ is the conformal time. Let an overhead circle denote a derivative with respect to the conformal time, $\stackrel{\circ}{f}=\mathrm{d} f / \mathrm{d} \eta$ for any $f$. We define the quantities $\mathcal{H}=\stackrel{a}{a} / a=$ $a H$ and $\bar{\Psi}=-\stackrel{\circ}{\rho}_{\Lambda} / \rho_{m}=a \Psi$, which are the analogues of $H$ and $\Psi$ in conformal time. Using the background local conservation equation (3) for the current epoch (neglecting therefore radiation) and rephrasing it in conformal time, i.e. $\stackrel{\circ}{\rho}_{\Lambda}+\stackrel{\circ}{\rho}_{m}+3 \mathcal{H} \rho_{m}=0$, we can bring (24) to

$\stackrel{\circ}{v}_{m}+\mathcal{H} v_{m}+\phi=\frac{\delta \rho_{\Lambda}}{\rho_{m}}-\bar{\Psi} v_{m}$

Once more the usual fluid equation (in this case Euler's equation) is retrieved if we arrange that $\delta \rho_{\Lambda}=\rho_{m} \bar{\Psi} v_{m}=a \rho_{m} \Psi v_{m}$, as then the two terms on the right-hand side of (25) cancel each other. Alternatively, one can use the covariant form $\nabla^{\mu} T_{\mu \nu}=Q_{\nu}$ for the local conservation law, with the source four-vector $Q_{\nu}=Q U_{v}$, where $U_{v}=(a, \mathbf{0})$ is the background matter four-velocity in conformal time. By perturbing the covariant conservation equation, one finds

$\delta\left(\nabla^{\mu} T_{\mu \nu}\right)=\delta Q_{v}=\delta Q U_{v}+Q \delta U_{v}$, 


\section{$w_{0}$}

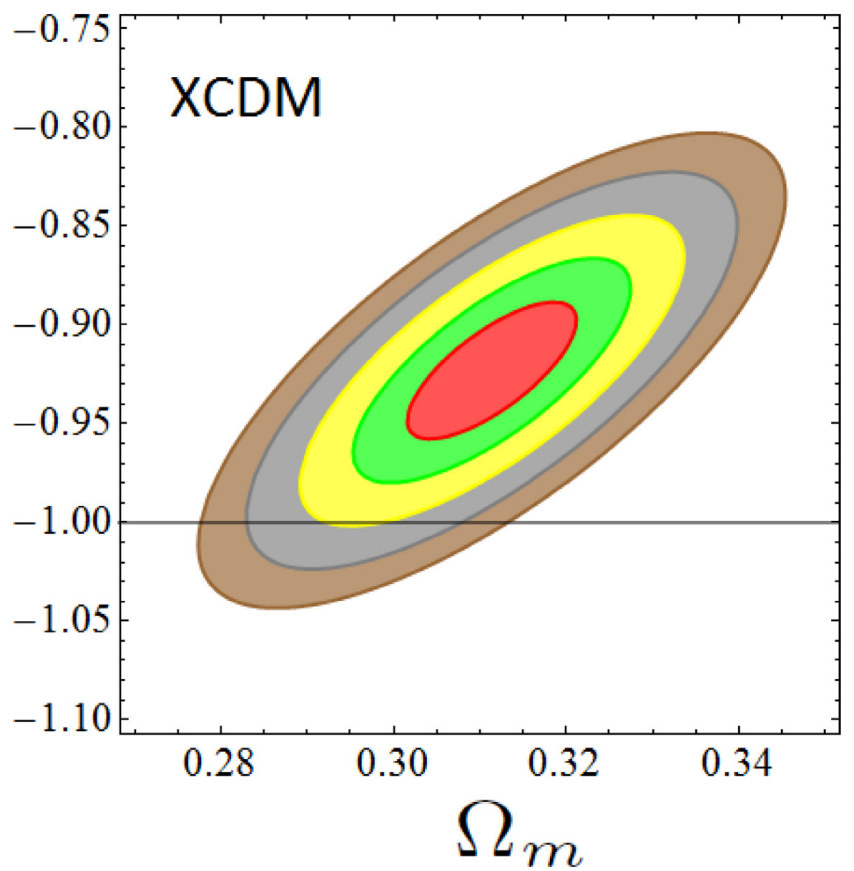

Figure 3. As in Fig. 1, but for model XCDM. The $\Lambda$ CDM is excluded in this case at $\sim 3 \sigma$ c.l. Marginalization over $\Omega_{\mathrm{m}}$ increases the c.l. up to $3.35 \sigma$ (cf. Table 1).

$\mathrm{f}(\mathrm{z}) \sigma_{\mathrm{B}}(\mathrm{z})$

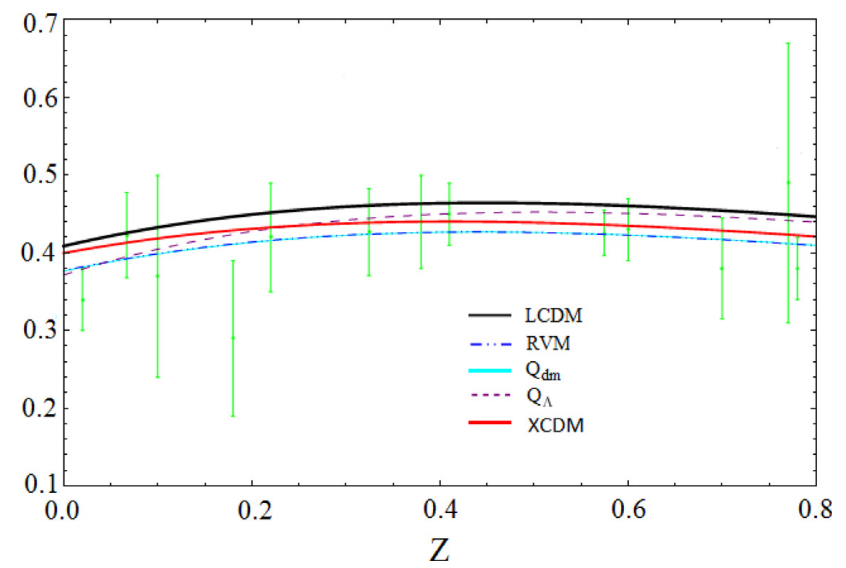

Figure 4. The $f(z) \sigma_{8}(z)$ data (cf. Section 3 ) and the predicted curves by the $\Lambda \mathrm{CDM}, \mathrm{XCDM}$, and the DVMs, for the best-fitting values in Table 1.

where $\delta Q$ and $\delta U_{v}=a(\phi,-v)$ are the perturbations of the source function and the four-velocity, respectively. Thus, we obtain

$\delta\left(\nabla^{\mu} T_{\mu \nu}\right)=a(\delta Q+Q \phi,-Q v)$.

From the $v=j$ component of the above equation, we derive anew the usual Euler equation $\stackrel{\circ}{m}_{m}+\mathcal{H} v_{m}+\phi=0$, which means that the relation $\delta \rho_{\Lambda}=a Q v_{m}=a \rho_{m} \Psi v_{m}$ is automatically fulfilled. So the analyses in the two gauges converge to the same final result for $\delta \rho_{\Lambda}$.
After we have found the condition that $\delta \rho_{\Lambda}$ must satisfy in each gauge so as to prevent that the vacuum modifies basic conservation laws of the matter fluid, one can readily show that any of the above equations (23) or (25) for each gauge (now with their right-hand side set to zero), in combination with the corresponding perturbed continuity equation and the perturbed 00-component of Einstein's equations (giving Poisson's equation in the Newtonian approximation), leads to the desired matter perturbations equation (22), in accordance with the result previously derived by other means in references Gómez-Valent et al. (2015a) and Basilakos \& Solà (2014). Altogether, the above considerations formulated in the context of different gauges allow us to consistently neglect the DE perturbations at scales down the horizon. This justifies the use of equation (22) for the effective matter perturbations equation in our study of linear structure formation in the framework of the DVMs. See Gómez-Valent \& Solà (2018) for an expanded exposition of these considerations.

For later convenience, let us also rewrite equation (22) in terms of the scale factor variable rather than the cosmic time. Using $\mathrm{d} / \mathrm{d} t=a H \mathrm{~d} / \mathrm{d} a$ and denoting the differentiation $\mathrm{d} / \mathrm{d} a$ with a prime, we find

$\delta_{m}^{\prime \prime}+\frac{A(a)}{a} \delta_{m}^{\prime}+\frac{B(a)}{a^{2}} \delta_{m}=0$,

where the functions $A$ and $B$ of the scale factor are given by

$$
\begin{aligned}
& A(a)=3+a \frac{H^{\prime}(a)}{H(a)}+\frac{\Psi(a)}{H(a)}, \\
& B(a)=-\frac{4 \pi G \rho_{m}(a)}{H^{2}(a)}+\frac{2 \Psi(a)}{H(a)}+a \frac{\Psi^{\prime}(a)}{H(a)} .
\end{aligned}
$$

\subsection{Initial conditions}

In order to solve (28) we have to fix appropriate initial conditions for $\delta_{m}(a)$ and $\delta_{m}^{\prime}(a)$ for each model at high redshift, say at $z_{i} \sim 100$ $\left(a_{i} \sim 10^{-2}\right.$ ), when non-relativistic matter dominates both over the vacuum and the radiation contributions. In practice, it can be fixed at lower redshifts, say of the order of 10 , where the subhorizon approximation is even more efficient (Gómez-Valent \& Solà 2018), although the differences are small. For small values of the scale factor, the normalized Hubble rate (squared) for each model, and the energy densities for the various components, see equations (10)-(18), can be significantly simplified. As a result we obtain the leading form of the functions (29) and (30) for the different DVMs:

RVM: $\quad A=\frac{3}{2}(1+3 v)$

$Q_{d m}: \quad A=\frac{3}{2}\left(1+v_{d m}\right)+3 \frac{\Omega_{d m}}{\Omega_{m}} v_{d m}+\mathcal{O}\left(v_{d m}^{2}\right)$

$\mathrm{Q}_{\Lambda}: \quad A=\frac{3}{2}$,

and

$\mathrm{RVM}: \quad B=-\frac{3}{2}+3 v+\frac{9}{2} v^{2}$

$Q_{d m}: \quad B=-\frac{3}{2}\left(1-v_{d m}-\frac{\Omega_{d m}}{\Omega_{m}} v_{d m}\right)+\mathcal{O}\left(v_{d m}^{2}\right)$

$Q_{\Lambda}: \quad B=-\frac{3}{2}$. 

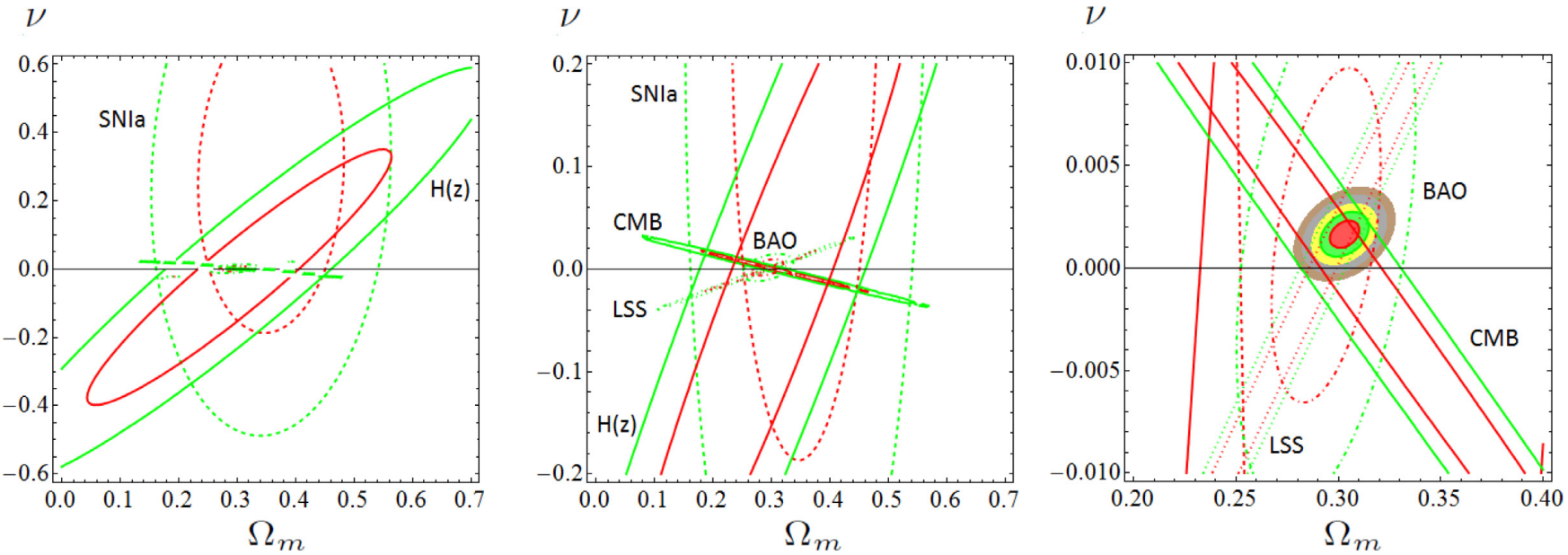

Figure 5. Reconstruction of the contour lines for the RVM, from the partial contour plots of the different SNIa+BAO+H(z)+LSS+CMB data sources. The $1 \sigma$ c.l. and $2 \sigma$ c.l. contours are shown in all cases. For the reconstructed final contour lines, we also plot the $3 \sigma, 4 \sigma$, and $5 \sigma$ confidence level regions.
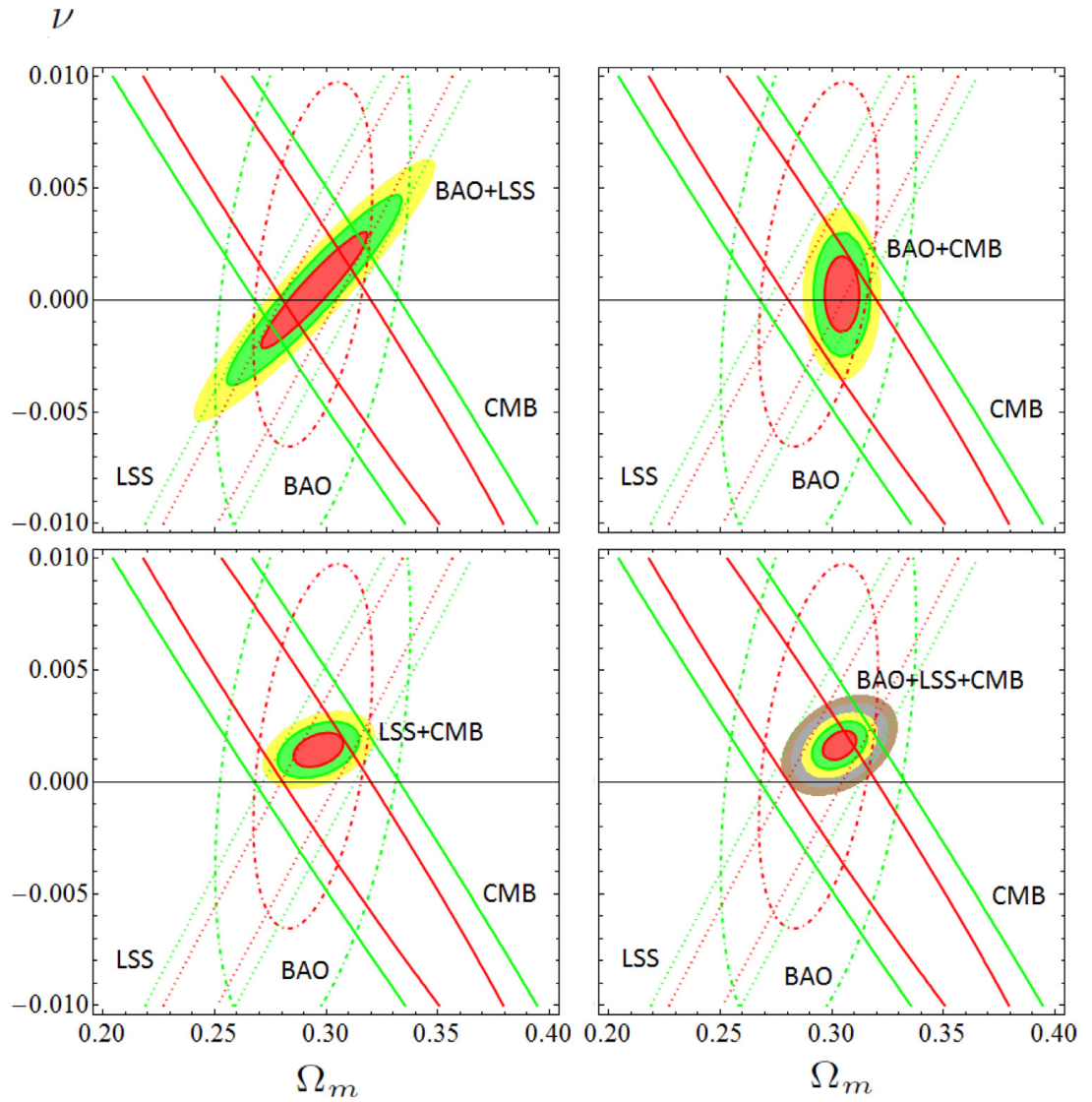

Figure 6. As in Fig. 5, but considering the effect of only the BAO, LSS, and CMB in all the possible combinations: BAO+LSS, BAO+CMB, LSS+CMB, and $\mathrm{BAO}+\mathrm{LSS}+\mathrm{CMB}$. As discussed in the text, it is only when such a triad of observables is combined that we can see a clear $\lesssim 4 \sigma \mathrm{c} .1$. effect, which is comparable to intersecting the whole set of SNIa+BAO+H(z)+LSS+CMB data.

For $v_{i} \rightarrow 0$, we recover the $\Lambda$ CDM behaviour $A \rightarrow \frac{3}{2}$ and $B \rightarrow-\frac{3}{2}$, as it should. This is already true for the $Q_{\Lambda}$ without imposing $v_{\Lambda} \rightarrow 0$, therefore its initial conditions are precisely the same as for the concordance model. Once the functions (29) and (30) take constant values (as it is the case here at the high redshifts where we fix the initial conditions), the differential equation (28) admits power-like solutions of the form $\delta_{m}\left(a_{i}\right)=a_{i}^{s}$. Of the two solutions, we are interested only in the growing mode solution, as this is the only one relevant for structure formation. For example, using (31) and (34) for the case of the RVM, the perturbations equation (28) 

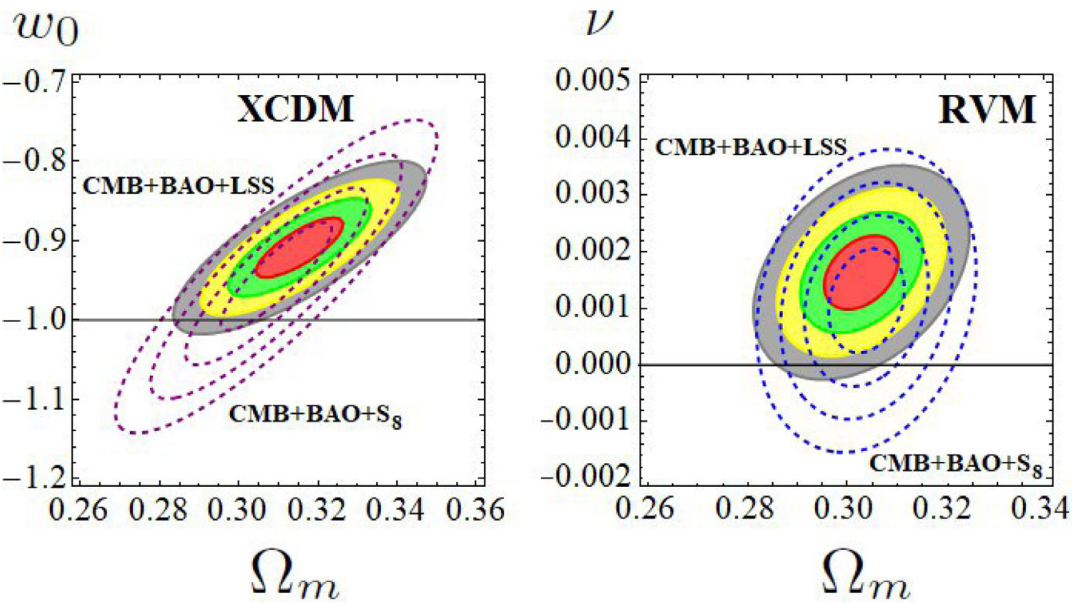

Figure 7. Contour lines for the XCDM (left) and RVM (right) using the same CMB+BAO+LSS data as in Table 1 (solid contours), and also when replacing the LSS data (i.e. the $f(z) \sigma_{8}(z)$ points) with the $S_{8}$ value obtained from the weak gravitational lensing data (Joudaki et al. 2018, dashed lines).

becomes

$\delta_{m}^{\prime \prime}+\frac{3}{2 a}(1+3 v) \delta_{m}^{\prime}-\left(\frac{3}{2}-3 v-\frac{9}{2} v^{2}\right) \frac{\delta_{m}}{a^{2}}=0$.

The power-law solution for the growing mode gives the result $\delta_{m}=a^{1-3 v}$, which is exact even keeping the $\mathcal{O}\left(v^{2}\right)$ term. Nevertheless, as warned previously, in practice we can neglect all $\mathcal{O}\left(v_{i}^{2}\right)$ contributions despite we indicate their presence. Repeating the same procedure for the other models, the power-law behaviour in each case for the growing mode solution $\delta_{m} \sim a^{s}$ is the following:

$\mathrm{RVM}: s=1-3 v$

$\mathrm{Q}_{\mathrm{dm}}: s=1-v_{d m}\left(\frac{6 \Omega_{m}+9 \Omega_{d m}}{5 \Omega_{m}}\right)+\mathcal{O}\left(v_{d m}^{2}\right)$

$\mathrm{Q}_{\Lambda}: s=1$.

Imposing the above analytical results to fix the initial conditions, we are then able to solve numerically the full differential equation (28) from a high redshift $z_{i} \sim 100\left(a_{i} \sim 10^{-2}\right)$ up to our days. The result does not significantly depend on the precise value of $z_{i}$, provided it is in the matter-dominated epoch and well below the decoupling time $\left(z \sim 10^{3}\right)$, where the radiation component starts to be nonnegligible.

\subsection{Linear growth and growth index}

The linear growth rate of clustering is an important (dimensionless) indicator of structure formation (Peebles 1993). It is defined as the logarithmic derivative of the linear growth factor $\delta_{m}(a)$ with respect to the $\log$ of the scale factor, $\ln a$. Therefore,

$f(a) \equiv \frac{a}{\delta_{m}} \frac{\mathrm{d} \delta_{m}}{\mathrm{~d} a}=\frac{\mathrm{d} \ln \delta_{m}}{\mathrm{~d} \ln a}$,

where $\delta_{m}(a)$ is obtained from solving the differential equation (28) for each model. The physical significance of $f(a)$ is that it determines the peculiar velocity flows (Peebles 1993). In terms of the redshift variable, we have $f(z)=-(1+z) \mathrm{d} \ln \delta_{m} / \mathrm{d} z$, and thus the linear growth can also be used to determine the amplitude of the redshift distortions. This quantity has been analytically computed for the RVM in Basilakos \& Solà (2015). Here, we shall take it into account for the study of the LSS data in our overall fit to the cosmological observations.
One usually expresses the linear growth rate of clustering in terms of $\Omega_{\mathrm{m}}(z)=\rho_{\mathrm{m}}(z) / \rho_{\mathrm{c}}(z)$, where $\rho_{\mathrm{c}}(z)=3 H^{2}(z) /(8 \pi G)$ is the evolving critical density, as follows (Peebles 1993):

$f(z) \simeq\left[\Omega_{\mathrm{m}}(z)\right]^{\gamma(z)}$,

where $\gamma$ is the so-called linear growth rate index. For the usual $\Lambda \mathrm{CDM}$ model, such an index is approximately given by $\gamma_{\Lambda} \simeq$ $6 / 11 \simeq 0.545$. For models with a slowly varying equation of state $w_{D}$ (i.e. approximately behaving as the XCDM, with $w_{D} \simeq w_{0}$ ), one finds the approximate formula $\gamma_{D} \simeq 3\left(w_{D}-1\right) /\left(6 w_{D}-5\right)$ (Wang \& Steinhardt 1998) for the asymptotic value when $\Omega_{\mathrm{m}} \rightarrow 1$. Setting $w_{D}=-1+\epsilon$, it can be rewritten

$\gamma_{D} \simeq \frac{6-3 \epsilon}{11-6 \epsilon} \simeq \frac{6}{11}\left(1+\frac{1}{22} \epsilon\right)$.

Obviously, for $\epsilon \rightarrow 0$ (equivalently, $\omega_{D} \rightarrow-1$ ) one retrieves the $\Lambda \mathrm{CDM}$ case. Since the current experimental error on the $\gamma$-index is of the order of 10 per cent, it opens the possibility to discriminate cosmological models using such an index (see e.g. Pouri, Basilakos \& Plionis 2014). In the case of the RVM and various models and frameworks, the function $\gamma(z)$ has been computed numerically in Gómez-Valent et al. (2015a). Under certain approximations, an analytical result can also be obtained for the asymptotic value (Basilakos \& Solà 2015):

$\gamma_{\mathrm{RVM}} \simeq \frac{6+3 v}{11-12 v} \simeq \frac{6}{11}\left(1+\frac{35}{22} v\right)$

This expression for the RVM is similar to (43) for an approximate $\mathrm{XCDM}$ parametrization, and it reduces to the $\Lambda \mathrm{CDM}$ value for $v=0$, as it should

\subsection{Weighted linear growth and power spectrum}

A most convenient observable to assess the performance of our vacuum models in regard to structure formation is the combined quantity $f(z) \sigma_{8}(z)$, viz. the ordinary growth rate weighted with $\sigma_{8}(z)$, the rms total matter fluctuation (baryons $+\mathrm{CDM}$ ) on $R_{8}=8 h^{-1} \mathrm{Mpc}$ spheres at the given redshift $z$, computed in linear theory. It has long been recognized that this estimator is almost a model-independent way of expressing the observed growth history of the Universe, most noticeably it is found to be independent of the galaxy density bias (Guzzo et al. 2008; Song \& Percival 2009). 
With the help of the above generalized matter perturbations (equation 28) and the appropriate initial conditions, the analysis of the linear LSS regime is implemented on using the weighted linear growth $f(z) \sigma_{8}(z)$. The variance of the smoothed linear density field on $R_{8}=8 h^{-1}$ Mpc spheres at redshift $z$ is computed from

$\sigma_{8}^{2}(z)=\delta_{m}^{2}(z) \int \frac{\mathrm{d}^{3} k}{(2 \pi)^{3}} P(k, \boldsymbol{p}) W^{2}\left(k R_{8}\right)$.

Here, $P(k, \boldsymbol{p})=P_{0} k^{n_{s}} T^{2}(k)$ is the ordinary linear matter power spectrum (i.e. the coefficient of the two-point correlator of the linear perturbations), with $P_{0}$ a normalization factor, $n_{s}$ the spectral index, and $T(k)$ the transfer function. Furthermore, $W\left(k R_{8}\right)$ in the above formula is a top-hat smoothing function (see e.g. Gómez-Valent et al. 2015a, for details), which can be expressed in terms of the spherical Bessel function of order 1, as follows:

$W\left(k R_{8}\right)=3 \frac{j_{1}\left(k R_{8}\right)}{k R_{8}}=\frac{3}{k^{2} R_{8}^{2}}\left(\frac{\sin \left(k R_{8}\right)}{k R_{8}}-\cos \left(k R_{8}\right)\right)$.

Moreover, $\boldsymbol{p}$ is the fitting vector with all the free parameters, including the specific vacuum parameters $v_{i}$ of the DVMs, or the EoS parameters $w_{i}$ for the XCDM/CPL parametrizations, as well as the standard parameters.

The power spectrum depends on all the components of the fitting vector. However, the dependence on the spectral index $n_{s}$ is power-like, whereas the transfer function $T(k, \boldsymbol{q})$ depends in a more complicated way on the rest of the fitting parameters (see below), and thus for convenience we collect them in the reduced fitting vector $\boldsymbol{q}$ not containing $n_{s}$. It is convenient to write the variance (45) in terms of the dimensionless linear matter power spectrum, $\mathcal{P}(k, \boldsymbol{p})=\left(k^{3} / 2 \pi^{2}\right) P(k, \boldsymbol{p})$, with

$\mathcal{P}(k, \boldsymbol{p})=\mathcal{P}_{0} k^{n_{s}+3} T^{2}(k, \boldsymbol{q})$.

The normalization factor $\mathcal{P}_{0}=P_{0} / 2 \pi^{2}$ will be determined in the next section in connection to the definition of the fiducial model.

For the transfer function, we have adopted the usual BBKS form (Bardeen et al. 1986), but we have checked that the use of the alternative one by Eisenstein \& Hu (1998) does not produce any significant change in our results. Recall that the wave number at equality, $k_{\text {eq }}$, enters the argument of the transfer function. However, $k_{\text {eq }}$ is a model-dependent quantity, which departs from the $\Lambda \mathrm{CDM}$ expression in those models in which matter and/or radiation are governed by an anomalous continuity equation, as e.g. in the DVMs. In point of fact $k_{\text {eq }}$ depends on all the parameters of the reduced fitting vector $\boldsymbol{q}$. For the concordance model, $k_{\mathrm{eq}}$ has the simplest expression,

$k_{\mathrm{eq}}^{\Lambda}=H_{0} \Omega_{\mathrm{m}} \sqrt{\frac{2}{\Omega_{\mathrm{r}}}}=\frac{\Omega_{\mathrm{m}} h^{2}}{2997.9} \sqrt{\frac{2}{\omega_{\mathrm{r}}}} \mathrm{Mpc}^{-1}$,

where $\omega_{r}=\Omega_{\mathrm{r}} h^{2}$. In the second equality, we have used the relation $H_{0}^{-1}=2997.9 h^{-1} \mathrm{Mpc}$. For the DVMs, it is not possible to find a formula as compact as the one above. Either the corresponding expression for $a_{\mathrm{eq}}$ is quite involved, as in the RVM case,

RVM: $\quad a_{\mathrm{eq}}=\left[\frac{\Omega_{\mathrm{r}}(1+7 v)}{\Omega_{\mathrm{m}}(1+3 v)+4 v \Omega_{\mathrm{r}}}\right]^{\frac{1}{1+3 v}}$,

or because $a_{\mathrm{eq}}$ must be computed numerically, as for the models $\mathrm{Q}_{d m}$ and $\mathrm{Q}_{\Lambda}$. In all cases, for $v_{i}=0$ we retrieve the value of $a_{\mathrm{eq}}$ in the $\Lambda \mathrm{CDM}$.

\subsection{Fiducial model}

Inserting the dimensionless power spectrum (47) into the variance (45) at $z=0$ allows us to write $\sigma_{8}(0)$ in terms of the power spectrum normalization factor $\mathcal{P}_{0}$ in (47) and the primary parameters that enter our fit for each model. This is tantamount to saying that $\mathcal{P}_{0}$ can be fixed as follows:

$\mathcal{P}_{0}=\frac{\sigma_{8, \Lambda}^{2}}{\delta_{m, \Lambda}^{2}}\left[\int_{0}^{\infty} k^{n_{s, \Lambda+3}} T^{2}\left(k, \boldsymbol{q}_{\Lambda}\right) W^{2}\left(k R_{8, \Lambda}\right)(\mathrm{d} k / k)\right]^{-1}$,

where the chosen values of the parameters in this expression define our fiducial model. The latter is characterized by the vectors of fiducial parameters $\boldsymbol{p}_{\Lambda}$ and $\boldsymbol{q}_{\Lambda}$, defined in obvious analogy with the original fitting vectors but with all their parameters taken to be equal to those from the Planck 2015 TT,TE,EE+lowP+lensing analysis (Planck collaboration XIII 2016), with $v_{i}=0$ for the DVMs and $w_{0}=-1, w_{1}=0$ for the XCDM/CPL parametrizations. The subindex $\Lambda$ carried by all the parameters denotes such a setting. In particular, $\sigma_{8, \Lambda} \equiv \sigma_{8, \Lambda}(0)$ in (50) is also taken from the aforementioned Planck 2015 data. However, $\delta_{m, \Lambda} \equiv \delta_{m, \Lambda}(0)$ in the same formula is computable: it is the value of $\delta_{m}(z=0)$ obtained from solving the perturbations equation of the $\Lambda \mathrm{CDM}$, using the mentioned fiducial values of the other parameters. Finally, plugging the normalization factor (50) in (45) and using (47) one finds

$\sigma_{8}(z)=\sigma_{8, \Lambda} \frac{\delta_{m}(z)}{\delta_{m, \Lambda}} \sqrt{\frac{\int_{0}^{\infty} k^{n_{s}+2} T^{2}(k, \boldsymbol{q}) W^{2}\left(k R_{8}\right) \mathrm{d} k}{\int_{0}^{\infty} k^{n_{s, \Lambda}+2} T^{2}\left(k, \boldsymbol{q}_{\Lambda}\right) W^{2}\left(k R_{8, \Lambda}\right) \mathrm{d} k}}$.

For the fiducial $\Lambda \mathrm{CDM}$, this expression just gives the scaling of $\sigma_{8, \Lambda}(z)$ with the redshift in the linear theory, that is to say, $\sigma_{8, \Lambda}(z) / \sigma_{8, \Lambda}=\delta_{m, \Lambda}(z) / \delta_{m, \Lambda}$. But for an arbitrary model, equation (51) normalizes the corresponding $\sigma_{8}(z)$ with respect to the fiducial value, $\sigma_{8, \Lambda}$. This includes, of course, our fitted $\Lambda \mathrm{CDM}$, which is not the same as the fiducial $\Lambda \mathrm{CDM}$. So all fitted models are compared to the same fiducial model defined by the Planck 2015 results. Similarly, upon computing with this method the weighted linear growth rate $f(z) \sigma_{8}(z)$ for each model under consideration (including the $\Lambda \mathrm{CDM}$ ), the functions $f(z) \sigma_{8}(z)$ for all models become normalized to the same fiducial model. It is important to emphasize that one cannot adjust the power spectrum and the $f(z) \sigma_{8}(z)$ values independently. Therefore, we first normalize with Planck 2015 results, as above described, and from here we fit the models to the data, in which the LSS component takes an essential part.

The connection of the normalization factor (50) with $A_{s}$ (Planck collaboration XIII 2016) can be easily found using standard formulae (Liddle \& Lyth 2000, 2009; Dodelson 2003). We find

$\mathcal{P}_{0}=\frac{4 A_{s}}{25} \frac{k_{*}^{1-n_{s}}}{H_{0}^{4} \Omega_{m}^{2}}$,

where $k_{*}=0.05 \mathrm{Mpc}^{-1}$ is the pivot scale used by Planck. This follows from the fact that $\mathcal{P}_{0}$ is related to $\delta_{H}^{2}$ (the primordial amplitude of the gravitational potential) through $\mathcal{P}_{0}=\delta_{H}^{2} /\left(H_{0}^{3+n_{s}} \Omega_{m}^{2}\right)$ and on the other hand we have $\delta_{H}^{2}=(4 / 25) A_{s}\left(H_{0} / k_{*}\right)^{n_{s}-1}$.

In Fig. 4, we display the theoretical results for $f(z) \sigma_{8}(z)$ from the various models, side by side with the LSS data measurements, using the fitted values of Table 1 . The values that we find for $\sigma_{8}(0)$ for each model, with the corresponding uncertainties, are reckoned in Table 1. Inspection of Fig. 4 shows that the DVMs provide a better description of the LSS data points as compared to the $\Lambda$ CDM. The $\mathrm{XCDM}$ parametrization takes an intermediate position, granting a better fit than the $\Lambda \mathrm{CDM}$ but a poorer one than the RVM and $Q_{d m}$. One can see that it is necessary an overall reduction of $\sim 8$ per cent in the value of $f(z) \sigma_{8}(z)$ with respect to the $\Lambda \mathrm{CDM}$ curve (the solid 
line on top of the others in that figure). Once $\Omega_{m}$ is accurately fixed from the $\mathrm{CMB}$ data, the $\Lambda \mathrm{CDM}$ model does not have any further freedom to further adjust the low- $z$ LSS data. This can be seen from equation (52) and from the fact that the normalization amplitude of the power spectrum $A_{s}$ as given by Planck tolerates an error of order 2 per cent at most (Planck collaboration XIII 2016), and therefore such residual freedom cannot be invested to adjust the structure formation data, it is simply insufficient as we have checked. Thus, there seems to be no way at present to describe correctly both CMB and LSS data within the $\Lambda \mathrm{CDM}$. This is of course at the root of the so-called $\sigma_{8}$-tension, one of the important problems of the $\Lambda \mathrm{CDM}$ mentioned in the introduction (see e.g. Macaulay, Wehus \& Eriksen 2013; Battye, Charnock \& Moss 2015; Basilakos \& Nesseris 2016, 2017, for additional discussion and references).

In contrast, the DVMs can provide a possible clue. For example, for the RVM case an analytical explanation has recently been provided in references Gómez-Valent \& Solà $(2017,2018)$ showing why the dynamical vacuum can help in relaxing such tension. Recall that for $v=0$ the equality point between matter and radiation as given in equation (49) boils down to the $\Lambda \mathrm{CDM}$ value. However, for $v \neq 0$ a non-negligible contribution is obtained, despite the smallness of $v$. Indeed, one can show that the $v$-effect causes a negative correction to the transfer function, which at linear order in $v$ is proportional to $6 v \ln \left(\Omega_{\mathrm{m}} / \Omega_{\mathrm{r}}\right) \simeq 50 \nu$. Since $v$ is fitted to be of the order of $\sim 10^{-3}$ in Table 1 , it follows that the aforementioned negative correction can easily enhance the final effect up to near 10 per cent level. Upon a careful analysis of all the contributions, it eventually amounts to a $\sim 8$ percent reduction of the weighted growth rate $f(z) \sigma_{8}(z)$ as compared to the $\Lambda \mathrm{CDM}$ value (Gómez-Valent \& Sol'a 2017, 2018). This is precisely the reduction with respect to the $\Lambda \mathrm{CDM}$ prediction that is necessary in order to provide a much better description of the LSS data, see Fig. 4. Interestingly enough, as a bonus one also obtains an excellent description of the current weak-lensing data; see Section 6.3.

\section{MAIN NUMERICAL RESULTS}

For the statistical analysis, we define the joint likelihood function as the product of the likelihoods for all the data sets. Correspondingly, for Gaussian errors the total $\chi^{2}$ to be minimized reads:

$\chi_{\mathrm{tot}}^{2}=\chi_{\mathrm{SNIa}}^{2}+\chi_{\mathrm{BAO}}^{2}+\chi_{H}^{2}+\chi_{\mathrm{LSS}}^{2}+\chi_{\mathrm{CMB}}^{2}$.

Each one of these terms is defined in the standard way and they include the corresponding covariance matrices.

Table 1 contains the main fitting results, whereas the other tables display complementary information. We observe from Fig. 1 that the vacuum parameters, $v$ and $v_{d m}$, are neatly projected non-null and positive for the RVM and the $Q_{d m}$. In the particular case of the RVM, Fig. 2 displays in a nutshell our main results in all possible planes of the fitting parameter space. Fig. 4, on the other hand, indicates that the XCDM is also sensitive to the DDE signal. In all cases, the LSS data play an important role (cf. Fig. 4). Focusing on the model that provides the best fit, namely the RVM, Figs 5 and 6 reveal the clue to the main data sources responsible for the final results. We will further comment on them in the next sections. Remarkably enough, the significance of this dynamical vacuum effect reaches up to about $\sim 3.8 \sigma$ c.l. after marginalizing over the remaining parameters.

\subsection{Fitting the data with the XCDM and CPL parametrizations}

Here, we further elaborate on the results we have found by exploring now the possible time evolution of the DE in terms of the wellknown XCDM and CPL parametrizations (introduced in Section 2.3). For the $\mathrm{XCDM}, w=w_{0}$ is the (constant) equation of state (EoS) parameter for X, whereas for the CPL there is also a dynamical component introduced by $w_{1}$, see equation (20). The corresponding fitting results for the XCDM parametrization is included in all our tables, along with those for the DVMs and the $\Lambda$ CDM. For the main Table 1, we also include the CPL fitting results. For example, reading off Table 1 we can see that the best-fitting value for $w_{0}$ in the XCDM is

$w_{0}=-0.923 \pm 0.023$.

It is worth noticing that this EoS value is far from being compatible with a rigid $\Lambda$-term. It actually departs from -1 by precisely $3.35 \sigma$ c.l. In Fig. 3, we depict the contour plot for the XCDM in the $\left(\Omega_{\mathrm{m}}\right.$, $w_{0}$ ) plane. Practically, all of the $3 \sigma$-region lies above the horizontal line at $w_{0}=-1$. Subsequent marginalization over $\Omega_{\mathrm{m}}$ renders the result (54). Concerning the CPL, we can see from Table 1 that the errors on the fitting parameters are larger, especially on $w_{1}$, but it concurs with the XCDM that DE dynamics is also preferred (see also Section 5.2).

Remarkably, from the rich string of $\mathrm{SNIa}+\mathrm{BAO}+H(z)+\mathrm{LSS}+\mathrm{CMB}$ data we find that even the simple XCDM parametrization is able to capture non-trivial signs of dynamical DE in the form of effective quintessence behaviour $\left(w_{0} \gtrsim-1\right)$, at more than $3 \sigma$ c.l. Given the significance of this fact, it is convenient to compare it with well-known previous fitting analyses of the XCDM parametrization, such as the ones performed by the Planck and BOSS collaborations 2-3 yr ago. The Planck 2015 value for the EoS parameter of the XCDM reads $w_{0}=-1.019_{-0.080}^{+0.075}$ (Planck collaboration XIII 2016) and the BOSS one is $w_{0}=-0.97 \pm 0.05$ (Aubourg et al. 2015). These results are perfectly compatible with our own fitting value for $w_{0}$ given in (54), but in stark contrast to it their errors are big enough as to be also fully compatible with the $\Lambda \mathrm{CDM}$ value $w_{0}=-1$. This is not too surprising if we bear in mind that none of these analyses included LSS formation data in their fits, as explicitly recognized in the text of their papers.

In the absence of the modern LSS data, we would indeed find a very different situation to that in Table 1 . As our Table 3 clearly shows that the removal of the LSS data set in our fit induces a significant increase in the magnitude of the central value of the EoS parameter for the XCDM, as well as of the corresponding error. This happens because the higher is $|w|$ the higher is the structure formation power predicted by the XCDM, and therefore the closer is such a prediction with that of the $\Lambda \mathrm{CDM}$ (which is seen to predict too much power as compared to the data, see Fig. 4). Under these conditions our analysis renders $w=-0.992 \pm 0.040$ (cf. Table 3), which is manifestly closer to (in fact consistent with) the aforementioned central values (and errors) obtained by Planck and BOSS teams. In addition, this result is now fully compatible with the $\Lambda$ CDM, as in the Planck 2015 and BOSS cases, and all of them are unfavoured by the LSS data.

From the foregoing observations, it becomes clear that in order to improve the fit to the observed values of $f(z) \sigma_{8}(z)$, which generally appear lower-powered with respect to those predicted by the $\Lambda \mathrm{CDM}$ (cf. Fig. 4), $|w|$ should decrease. This is just what happens in our fit for the XCDM, see equation (54). At the level of the DVMs this 
translates into positive values of $v_{i}$, as these values cause the vacuum energy to be larger in our past; and, consequently, it introduces a time modulation of the growth suppression of matter. It is apparent from Fig. 4 that the $f(z) \sigma_{8}(z)$ curves for the vacuum models are shifted downwards (they have less power than the $\Lambda \mathrm{CDM}$ ), and hence adapt significantly better to the LSS data points.

\subsection{Comparing the competing vacuum models through Akaike and Bayesian information criteria}

We may judge the fit quality obtained for the different vacuum models in this work from a different perspective. Although the $\chi_{\min }^{2}$ value of the overall fits for the main DVMs (RVM and $Q_{d m}$ ) and XCDM appear to be definitely smaller than the $\Lambda \mathrm{CDM}$ one, it proves extremely useful to reassess the degree of success of each competing model by invoking the time-honoured Akaike and Bayesian information criteria, denoted as AIC and BIC (Akaike 1974; Schwarz 1978; Kass \& Raftery 1995). The Akaike information criterion is defined as follows:

$\mathrm{AIC}=\chi_{\min }^{2}+\frac{2 n N}{N-n-1}$,

whereas the Bayesian information criterion reads

$\mathrm{BIC}=\chi_{\min }^{2}+n \ln N$.

In these formulas, $n$ is the number of independent fitting parameters and $N$ is the number of data points. The added terms on $\chi_{\min }^{2}$ represent the penalty assigned by these information criteria to the models owing to the presence of additional parameters. To test the degree of success of a dynamical DE model (versus the $\Lambda \mathrm{CDM}$ ) with the information criteria, we have to evaluate the pairwise differences $\triangle \mathrm{AIC}(\triangle \mathrm{BIC})$ between the AIC and BIC values of the $\Lambda \mathrm{CDM}$ with respect to the corresponding values of the models having a smaller value of these criteria - in our case the DVMs, XCDM, and CPL. The larger these (positive) differences are the higher is the evidence against the model with larger value of AIC (BIC) - i.e. the $\Lambda$ CDM in the present case.

According to the standard usage, for $\triangle \mathrm{AIC}$ and/or $\triangle \mathrm{BIC}$ below 2 one judges that there is 'consistency' between the two models under comparison; in the range 2-6, there exists a 'positive evidence' in favour of the model with smaller value of AIC and/or BIC; for values within 6-10, one may claim 'strong evidence' in favour of such a model; finally, above 10 , one speaks of 'very strong evidence'. The evidence ratio associated to acceptance of the favoured model and rejection of the unfavoured model is given by the ratio of Akaike weights, $A \equiv e^{\triangle \mathrm{AIC} / 2}$. Similarly, $B \equiv e^{\triangle \mathrm{BIC} / 2}$ estimates the so-called Bayes factor, which gives the ratio of marginal likelihoods between the two models (Amendola 2015; Amendola \& Tsujikawa 2015). Table 1 reveals conspicuously that the $\Lambda \mathrm{CDM}$ appears disfavoured when confronted to the DDE models. The most favoured one is the RVM, followed by the $Q_{d m}$ and next by the XCDM. In the case of CPL and $Q_{\Lambda}$, the improvement is only mild.

The AIC and BIC criteria can be thought of as a modern quantitative formulation of Occam's razor, in which the presence of extra parameters in a given model is conveniently penalized so as to achieve a fairer comparison with the model having less parameters.

\section{DISCUSSION}

In this section, we consider in more detail some important aspects and applications of our analysis. In particular, we identify which are the most important data sources which are responsible for the possible DDE signal and show that in the absence of any of these important ingredients the signal becomes weakened or completely inaccessible.

\subsection{Testing the impact of the different data sets in our analysis} and comparing with Planck 2015

The current work follows the track of Solà, Gómez-Valent \& de Cruz Pérez (2015) and is also firmly aligned with Solà, Gómez-Valent \& de Cruz Pérez (2017a) and Solà, de Cruz Pérez \& Gómez-Valent (2018). Although the models analysed in Solà, Gómez-Valent \& de Cruz Pérez $(2015,2017$ a) have some differences with respect to the ones treated here, the outcome of the analysis points to the very same direction, to wit that some DVMs and the XCDM fit better the available data than the $\Lambda \mathrm{CDM}$. But we want to emphasize some important aspects of the analysis carried out in this paper as compared to other analyses:

(i) We have used a large and fully updated set of cosmological $\mathrm{SNIa}+\mathrm{BAO}+H(z)+\mathrm{LSS}+\mathrm{CMB}$ observations. To our knowledge, this is one of the most complete and consistent data sets used in the literature, see Solà, Gómez-Valent \& de Cruz Pérez (2017a) up to some updating introduced here, especially concerning the LSS data.

(ii) We have removed all data that would entail double counting and used the known covariance matrices in the literature. As an example, we have avoided to use Hubble parameter data extracted from $\mathrm{BAO}$ measurements, and restricted only to those based on the differential age (i.e. the cosmic chronometers).

(iii) We have duly taken into account all the known covariance matrices in the total $\chi^{2}$-function (53), which means that we have accounted for all the known correlations among the data. Not all data sets existing in the literature are fully consistent, sometimes they are affected from important correlations that have not been evaluated. We have discussed the consistency of the present data in Solà, Gómez-Valent \& de Cruz Pérez (2017a).

We have conducted several practical tests in order to study the influence of different data sets in our fitting analysis. As previously mentioned, we have checked what is the impact on our results if we omit the use of the LSS data (cf. Table 3), but in our study we have also assessed what happens if we disregard the CMB data (cf. Table 4) while still keeping all the remaining observations. The purpose of this test is to illustrate once more the inherent $\sigma_{8}$-tension existing between the geometry data and the structure formation data. In both cases, namely when we dispense with the LSS or the CMB data, we find that for all the models under study the error bars for the fitted DDE parameters $\left(w_{i}, v_{i}\right)$ become critically larger (sometimes they increase a factor 2-4) than those displayed in Table 1, and as a consequence these parameters become fully compatible with the $\Lambda \mathrm{CDM}$ values (in particular $v_{i}=0$ for the DVMs) within $1 \sigma$ c.l. or less, which is tantamount to saying that the DDE effect is washed out. At the same time, and in full accordance with the mentioned results, the $\triangle \mathrm{AIC}$ and $\triangle \mathrm{BIC}$ information criteria become negative, which means (according to our definition in Section 5.2) that none of these DDE models fits better the data than the $\Lambda$ CDM under these particular conditions. These facts provide incontestable evidence of the strong constraining power of the LSS as well as of the CMB data, whether taken individually or in combination, and of their capability for narrowing down the allowed region in the parameter space. In the absence of either one of them, the $\Lambda$ CDM model is preferred over the DDE models, but only at the expense of ignoring the CMB input, or the LSS data, both of which are of course of utmost importance. Thus, the concordance model is now able to 
fit the LSS data better only because it became free from the tight CMB constraint on $\Omega_{\mathrm{m}}$, which enforced the latter to acquire a larger value. Without such constraint, a lower $\Omega_{\mathrm{m}}$ value can be chosen by the fitting procedure, what in turn enhances the agreement with the $f(z) \sigma_{8}(z)$ data points. We have indeed checked that the reduction of $\Omega_{\mathrm{m}}$ in the $\Lambda \mathrm{CDM}$ directly translates into an 8.6 percent lowering of $\sigma_{8}(0)$ with respect to the value shown in Table 1 for this model, namely we find that $\sigma_{8}(0)$ changes from $0.801 \pm 0.009$ (as indicated in Table 1) to $0.731 \pm 0.019$ when the CMB data are not used. Such substantial decrease tends to optimize the fit of the LSS data, but only at the expense of ruining the fit to the CMB when these data are restored. This is, of course, the very meaning of the $\sigma_{8}$-tension, which cannot be overcome at the moment within the $\Lambda \mathrm{CDM}$.

In stark contrast with the situation in the $\Lambda \mathrm{CDM}$, when the vacuum is allowed to acquire a mild dynamical component the $\sigma_{8-}$ tension can be dramatically loosened; see Gómez-Valent \& Solà $(2017,2018)$ for a detailed explanation. This can be seen immediately on comparing the current auxiliary tables 3 and 4 with the main Table 1. Recall that a positive vacuum energy suppresses the growth of structure formation and this is one of the reasons why the $\Lambda \mathrm{CDM}$ model is highly preferred to the CDM with $\Lambda=0$. Similarly, but at a finer and subtler level of precision, a time modulation of the growth suppression through dynamical vacuum energy or in general DDE should further help in improving the adjustment of the LSS data. In our case, this is accomplished e.g. by the $v$-parameter of the RVM, which enables a dynamical modulation of the growth suppression through the $\sim v H^{2}$ component of the vacuum energy density - cf. equation (8). The presence of this extra degree of freedom allows the DVMs to better adjust the LSS data without perturbing the requirements from the $\mathrm{CMB}$ data (which can therefore preserve the standard $\Omega_{\mathrm{m}}$ value obtained by Planck 2015). The fact that this readjustment of the LSS data by a dynamical component in the vacuum energy is possible is because the epoch of structure formation is very close to the epoch when the DE starts to dominate, which is far away from the epoch when the CMB was released, and hence any new feature of the DE can play a significant role in the LSS formation epoch without disrupting the main features of the CMB. Let us recall at this point that the presence of the extra parameter from the DDE models under discussion is conveniently penalized by the Akaike and Bayesian information criteria in our analysis, and thus the DDE models appear to produce a better fit than the $\Lambda \mathrm{CDM}$ under perfectly fair conditions of statistical comparison between competing models describing the same data.

The conclusion of our analysis is clear: no signal of DDE can be found without the inclusion of the CMB data and/or the LSS data, even keeping the rest of observables within the fit. Both the LSS and CMB data are crucial ingredients to enable capturing the DDE effect, and the presence of BAO data just enhances it further. This conclusion is additionally confirmed by our study of the deconstruction and reconstruction of the RVM contour plots in Figs 5 and 6 and is discussed at length in the next section.

We close this section by answering a most natural question. Why the dynamical DE signal that we are glimpsing here escaped undetected from the fitting analyses of Planck 2015? The answer can be obtained by repeating our fitting procedure and restricting ourselves to the much more limited data sets used by the Planck 2015 collaboration, more precisely in the papers (Planck collaboration XIII 2016; Planck collaboration XIV 2016). In contrast to Planck collaboration XIII (2016), where no LSS (RSD) data were used, in the case of Planck collaboration XIV (2016) they used only some BAO and LSS data, but their fit is rather limited in scope. Specifically, they used only four BAO data points, one AP (Alcock-Paczynski parameter) data point, and one single LSS point, namely the value of $f(z) \sigma_{8}(z)$ at $z=0.57$ - see details in that paper. Using this same data, we obtain the fitting results presented in our Table 5. They are perfectly compatible with the fitting results mentioned in Section 5.1 obtained by Planck 2015 and BOSS (Aubourg et al. 2015), i.e. none of them carries evidence of dynamical DE, with only the data used by these collaborations 2-3 yr ago.

In contradistinction to them, in our full analysis presented in Table 1 we used 11 BAO and 13 LSS data points, some of them available only from the recent literature and of high precision (Gil-Marín et al. 2017). From Table 5, it is apparent that with only the data used in Planck collaboration XIV (2016) the fitting results for the RVM are poor enough and cannot still detect clear traces of the vacuum dynamics. In fact, the vacuum parameters are compatible with zero at $1 \sigma \mathrm{c} .1$. and the values of $\triangle \mathrm{AIC}$ and $\triangle \mathrm{BIC}$ in that table are moderately negative, showing that the DVMs do not fit better the data than the $\Lambda$ CDM model with only such a limited input. In fact, not even the XCDM parametrization is capable of detecting any trace of dynamical DE with that limited data set, as the effective EoS parameter is compatible with $w_{0}=-1$ at roughly $1 \sigma$ c.l. $\left(w_{0}=-0.961 \pm 0.033\right)$.

The features that we are reporting here have remained hitherto unnoticed in the literature, except in Solà, Gómez-Valent \& de Cruz Pérez (2015, 2017a,b,c,d), and in Zhao et al. (2017). In the last reference, the authors have been able to find a significant $3.5 \sigma$ c.l. effect on dynamical DE, presumably in a model-independent way and following a non-parametric procedure, see also (Wang et al. 2015). The result of (Zhao et al. 2017) is well along the lines of this work.

\subsection{Deconstruction and reconstruction of the RVM contour plots}

We further complement our analysis by displaying in a graphical way the deconstructed contributions from the different data sets to our final contour plots in Fig. 1, for the specific case of the RVM. One can do similarly for any of the models under consideration. The result is depicted in Fig. 5, where we can assess the detailed deconstruction of the final contours in terms of the partial contours from the different $\mathrm{SNIa}+\mathrm{BAO}+H(z)+\mathrm{LSS}+\mathrm{CMB}$ data sources.

The deconstruction plot for the RVM case is dealt with in Fig. 5, through a series of three plots made at different magnifications. In the third plot of the sequence we can immediately appraise that the $\mathrm{BAO}+\mathrm{LSS}+\mathrm{CMB}$ data subset plays a fundamental role in narrowing down the final physical region of the $\left(\Omega_{\mathrm{m}}, v\right)$ parameter space, in which all the remaining parameters have been marginalized over. This deconstruction process also explains in very transparent visual terms why the conclusions that we are presenting here hinge to a large extent on some particularly sensitive components of the data. While there is no doubt that the CMB is a high-precision component in the fit, our study demonstrates (both numerically and graphically) that the maximum power of the fit is achieved when it is combined with the wealth of BAO and LSS data points currently available.

To gauge the importance of the $\mathrm{BAO}+\mathrm{LSS}+\mathrm{CMB}$ combination more deeply, in Fig. 6 we try to reconstruct the final RVM plot in Fig. 1 (left) from only these three data sources. First, we consider the overlapping regions obtained when we cross the pairs of data sources $\mathrm{BAO}+\mathrm{LSS}, \mathrm{BAO}+\mathrm{CMB}, \mathrm{LSS}+\mathrm{CMB}$ and finally the trio $\mathrm{BAO}+\mathrm{LSS}+\mathrm{CMB}$ (in all cases excluding the SNIa and $H(z)$ data). One can see that neither the BAO+LSS nor the BAO+CMB crossings yield a definite sign for $v$. This is consistent with the numerical results in Tables 3 and 4, where the removal of the LSS and 
the CMB data, respectively, renders rather poor fits with negative values of $\triangle \mathrm{AIC}$ and $\triangle \mathrm{BIC}$.

Remarkably, it is the LSS+CMB combination the one that carries a well-defined, positive, sign for $v$, as it is seen from the lower-left plot in Fig. 6, where $\triangle \mathrm{AIC}$ and $\triangle \mathrm{BIC}$ are now both positive and above 6 for the main DVMs (RVM and $Q_{d m}$ ), as we have checked. Finally, when we next intersect the pair LSS+CMB with the BAO data the signal peaks at $3.8 \sigma$ c.l., the final contours being now those shown in the lower-right plot of Fig. 6 . The outcome of this exercise is clear. For the RVM case, we have checked that the final BAO+LSS+CMB plot in Fig. 6 is essentially the same as the original RVM plot in Fig. 1 (the leftmost one). In other words, the final RVM contour plot containing the information from all our five data sources can essentially be reconstructed with only the triad of leading observables $\mathrm{BAO}+\mathrm{LSS}+\mathrm{CMB}$.

\subsection{Vacuum dynamics, structure formation, and weak-lensing data}

Owing to the significant role played by the structure formation data in the extraction of the possible DDE signal, we next inquire into its impact when we use a different proxy to describe such data. Let us note that an account of the LSS observations does not only concern the $f(z) \sigma_{8}(z)$ data, but also the weak gravitational lensing constraints existing in the literature on the conventional quantity $S_{8} \equiv \sigma_{8}\left(\Omega_{\mathrm{m}} / 0.3\right)^{0.5}$ (Heymans et al. 2013; Hildebrandt et al. 2017; Joudaki et al. 2018). In Fig. 7, we compare the respective results that we find for the XCDM (left) and the RVM (right) when we use either the $\mathrm{CMB}+\mathrm{BAO}+f \sigma_{8}$ or the $\mathrm{CMB}+\mathrm{BAO}+S_{8}$ data sources. For definiteness we use the recent study by Joudaki et al. (2018), in which they carry a combined analysis of cosmic shear tomography, galaxy-galaxy lensing tomography, and redshift-space multipole power spectra using imaging data by the Kilo Degree Survey (KiDS-450) overlapping with the 2-degree Field Lensing Survey (2dFLenS) and the Baryon Oscillation Spectroscopic Survey (BOSS). They find $S_{8}=0.742 \pm 0.035$. Incidentally, this value is $2.6 \sigma$ below the one provided by Planck's TT+lowP analysis [4]. Very similar results can be obtained using the weak gravitational lensing tomography data by KiDS-450 collaboration, $S_{8}=0.745 \pm 0.039$ (Hildebrandt et al. 2017), and also by CFHTLenS, $\left(\Omega_{\mathrm{m}} / 0.27\right)^{0.46}=0.770 \pm 0.040$ (Heymans et al. 2013). In contrast, the result $S_{8}=0.783_{-0.025}^{+0.021}$ provided by DES (DES collaboration 2017) is more resonant with Planck, but due to its large uncertainty it is still fully compatible with Joudaki et al. (2018), Hildebrandt et al. (2017), and Heymans et al. (2013). From Fig. 7, we confirm (using both the XCDM and the RVM) that the contour lines computed from the data string $\mathrm{CMB}+\mathrm{BAO}+f \sigma_{8}$ are mostly contained within the contour lines from the alternative string $\mathrm{CMB}+\mathrm{BAO}+\mathrm{S}_{8}$ and are shifted upwards. The former data set is therefore more precise and capable of resolving the DDE signal at a level of more than $3 \sigma$, whereas with $S_{8}$ it barely surpasses the $1 \sigma$ c.l. within the RVM and even less with the XCDM, thus rendering essentially no DDE signal. The outcome of this additional test is that the use of the weak-lensing data from $S_{8}$ as a replacement for the direct LSS measurements $\left(f \sigma_{8}\right)$ is insufficient since it definitely weakens the evidence in favour of DDE.

\section{CONCLUSIONS}

To conclude, in this work we aimed at testing cosmological physics beyond the standard or concordance $\Lambda \mathrm{CDM}$ model, which is built upon a rigid cosmological constant. We have presented a comprehensive study on the possibility that the global cosmological observations can be better described in terms of vacuum models equipped with a dynamical component that evolves with the cosmic expansion. This should be considered a natural possibility in the context of QFT in a curved background. Our task focused on three DVMs: RVM along with two more phenomenological models, denoted $Q_{d m}$ and $Q_{\Lambda}-$ see Section 2 .

At the same time, we have compared the performance of these models with the general XCDM and CPL parametrizations. We have fitted all these models and parametrizations to the same set of cosmological data based on the observables $\mathrm{SNIa}+\mathrm{BAO}+H(z)+\mathrm{LSS}+\mathrm{CMB}$. The remarkable outcome of this investigation is that in all the considered cases we find an improvement of the description of the cosmological data in comparison to the $\Lambda \mathrm{CDM}$.

The 'deconstruction analysis' of the contour plots in Section 6.2 has revealed which are the most decisive data ingredients responsible for the dynamical vacuum signal. We have identified that the $\mathrm{BAO}+\mathrm{LSS}+\mathrm{CMB}$ components play a momentous role in the overall fit, as they are responsible for the main effects uncovered here. The impact of the SNIa and $H(z)$ observables appears to be more moderate. While the SNIa data were of course essential for the detection of a non-vanishing value of $\Lambda$, these data do not seem to have sufficient sensitivity (at present) for the next-to-leading step, which is to unveil the possible dynamics of $\Lambda$. The sensitivity for that seems to be reserved for the LSS, BAO, and CMB data.

We have also found that the possible signs of DDE tend to favour an effective quintessence behaviour, in which the energy density decreases with the expansion. Whether or not the ultimate reason for such a signal stems from the properties of the quantum vacuum or from some particular quintessence model, it is difficult to say at this point. Quantitatively, the best fit is granted in terms of the RVM. The results are consistent with the traces of DDE that can also be hinted at with the help of the XCDM and CPL parametrizations.

In our work we have also clarified why previous fitting analyses based e.g. on the simple XCDM parametrization, such as the ones by the Planck 2015 (Planck collaboration XIII 2015; Planck collaboration XIV 2015) and BOSS collaborations (Aubourg et al. 2015), missed the DDE signature. Basically, the reason stems from not using a sufficiently rich sample of the most crucial data, namely BAO and LSS, some of which were unavailable a few years ago, and could not be subsequently combined with the CMB data.

More recently, signs of DDE at $\sim 3.5 \sigma$ c.l. have been reported from non-parametric studies of the observational data on the DE, which aim at a model-independent result (Zhao et al. 2017). The findings of their analysis are compatible with the ones we have reported here. Needless to say, statistical evidence conventionally starts at $5 \sigma$ c.l. and we will have to wait for updated observations to see if such a level of significance can be achieved in the future.

\section{ACKNOWLEDGEMENTS}

We are partially supported by MINECO FPA2016-76005-C2-1-P, 2017-SGR-929 (Generalitat de Catalunya), and MDM-2014-0369 (ICCUB). JS acknowledges the hospitality and support received from the Institute for Advanced Study at the Nanyang Technological University in Singapore while part of this work was being accomplished. 


\section{REFERENCES}

Abbott L., 1985, Phys. Lett. B, 150, 427

Akaike H., 1974, IEEE Trans. Autom. Control, 19, 716

Amendola L., 2000, Phys. Rev. D, 62, 043511

Amendola L., 2015, Notes for the course Statistical Physics. Heidelberg Univ

Amendola L., Tsujikawa S., 2015, Dark Energy. Theory and Observations. Cambridge Univ. Press, Cambridge

Aubourg E. et al., 2015, Phys. Rev. D, 92, 123516

Babić A., Guberina B., Horvat R., Štefančić H., 2005, Phys. Rev. D, 71, 124041

Bardeen J. M., Bond J. R., Kaiser N., Szalay A. S., 1986, ApJ, 304, 15

Barr S. M., 1987, Phys. Rev. D, 36, 1691

Barr S. M., Hochberg D., 1988, Phys. Lett. B, 211, 49

Basilakos S., 2015, Mod. Phys. Lett. A, 30, 1540031

Basilakos S., Nesseris S., 2016, Phys. Rev. D, 94, 123525

Basilakos S., Nesseris S., 2017, Phys. Rev. D, 96, 063517

Basilakos S., Solà J., 2014, Phys. Rev. D, 90, 023008

Basilakos S., Solà J., 2015, Phys. Rev. D, 92, 123501

Basilakos S., Plionis M., Solà J., 2009, Phys. Rev. D, 80, 083511

Basilakos S., Polarski D., Solà J., 2012, Phys. Rev. D, 86, 043010

Battye R. A., Charnock T., Moss A., 2015, Phys. Rev. D, 91, 103508

Betoule M. et al., 2014, A\&A, 568, 22

Beutler F. et al., 2011, MNRAS, 416, 3017

Beutler F. et al., 2012, MNRAS., 423, 3430

Blake C. et al., 2011, MNRAS, 415, 2876

Blake C. et al., 2013, MNRAS, 436, 3089

Bolotin Y. L., Kostenko A., Lemets O. A., Yerokhin D. A., 2015, Int. J. Mod. Phys. D, 24, 1530007

Caldwell R. R., Dave R., Steinhardt P. J., 1998, Phys. Rev. Lett., 80, 1582

Caldwell R. R., Kamionkowski M., Weinberg N. N., 2003, Phys. Rev. Lett., 91, 071301

Chen Y., Ratra B., Biesiada M., Li S., Zhu Z.-H, 2016, ApJ, 829, 61

Chevallier M., Polarski D., 2001, Int. J. Mod. Phys. D, 10, 213

Copeland E. J., Sami M., Tsujikawa S., 2006, Int. J. Mod. Phys. D, 15, V 1753

Costa A. A., Xu X. D., Wang B., Abdalla E., 2017, J. Cosmol. Astropart. Phys., 1701, 028

Delubac T. et al., 2015, A\&A, 574, A59

DES Collaboration, 2017, Dark Energy Survey Year 1 Results: Cosmological Constraints from Galaxy Clustering and Weak Lensing, preprint (arXiv:1708.01530)

Dodelson S., 2003, Modern Cosmology. Academic Press, New York

Dolgov A. D., 1983, in Gibbons G., Hawking S. W., Tiklos S. T., eds, The very Early Universe. Cambridge Univ. Press, Cambridge, p. 449.

Einstein A., 1917, Kosmologische Betrachtungen zur allgemeinen Relativitätstheorie, Sitzungsber. Königl. Preuss. Akad. Wiss. phys.-math. Klasse VI 142 (Submitted: February 8th, 1917)

Eisenstein D. J., Hu W., 1998, ApJ, 496, 605

Endo M., Fukui T., 1977, Gen. Relativ. Gravit., 8, 833

Endo M., Fukui T., 1982, Gen. Relativ. Gravit., 14, 769

España-Bonet C., Ruiz-Lapuente P., Solà J., Shapiro I. L., 2004, J. Cosmol. Astropart. Phys., 0402, 006,

Feix M., Nusser A., Branchini E., 2015, Phys. Rev. Lett., 115, 011301

Ford L. H., 1987, Phys. Rev. D, 35, 2339

Freedman W. L., 2017, Nat. Astron., 1, 0169

Fritzsch H., Solà J., 2012, Class. Quantum Gravity, 29, 215002

Fritzsch H., Solà J., 2015, Mod. Phys. Lett. A, 30, 1540034

Fritzsch H., Solà J., Nunes R. C., 2017, Eur. Phys. J. C, 77, 193

Fujii Y., 1982, Phys. Rev. D, 26, 2580

Geng C. Q., Lee C. C., Zhang K., 2016, Phys. Lett. B, 760, 422

Geng C.-Q., Lee C.-C., Yin L., 2017, J. Cosmol. Astropart. Phys., 1708, 032

Gil-Marín H. et al., 2017, MNRAS, 465, 1757

Gómez-Valent A., Solà J., 2015, MNRAS, 448, 2810

Gómez-Valent A., Solà J., 2017, Europhys. Lett., 120, 39001

Gómez-Valent A., Solà J., 2018, MNRAS, 478, 126,
Gómez-Valent A., Solà J., Basilakos S., 2015a, J. Cosmol. Astropart. Phys., 1501,004

Gómez-Valent A., Karimkhani E., Solà J., 2015b, J. Cosmol. Astropart. Phys., 1512, 048

Grande J., Pelinson A., Solà J., 2009, Phys. Rev. D, 79, 043006

Grande J., Solà J., Basilakos S., Plionis M., 2011, J. Cosmol. Astropart. Phys., 1108, 007

Granett B. R. et al., 2015, A\&A, 583, A61

Guo R. Y., Zhang J.-F., Zhang X., 2018, Exploring neutrino mass and mass hierarchy in the scenario of vacuum energy interacting with cold dark matter, preprint (arXiv:1803.06910)

Guzzo L. et al., 2008, Nature, 451, 541

Heymans C. et al., 2013, MNRAS, 432, 2433

Hildebrandt H. et al., 2017, MNRAS, 465, 1454

Jiménez R., Verde L., Treu T., Stern D., 2003, ApJ, 593, 622

Joudaki S. et al., 2018, MNRAS, 474, 4894

Kass R. E., Raftery A., Amer J., 1995, Stat. Assoc., 90, 773

Kazin E. A. et al., 2014, MNRAS, 441, 3524

Li Y. H., Zhang J. F., Zhang X., 2016, Phys. Rev. D, 93, 023002

Liddle A. R., Lyth D. H., 2000, Cosmological Inflation and Large-Scale Structure. Cambridge Univ. Press, Cambridge

Liddle A. R., Lyth D. H., 2009, The Primordial Density Perturbation. Cambridge Univ. Press, Cambridge

Lima J. A. S., 1996, Phys. Rev. D, 54, 2571

Lima J. A. S., Basilakos S., Solà J., 2013, MNRAS, 431, 923

Lima J. A. S., Basilakos S., Solà J., 2015, Gen. Relativ. Gravit., 47, 40

Lima J. A. S., Basilakos S., Solà J., 2016, Eur. Phys. J. C, 76, 228

Linder E. V., 2003, Phys. Rev. Lett., 90, 091301

Linder E. V., 2004, Phys. Rev. D, 70, 023511

Ma C. P., Bertschinger E., 1995. ApJ, 455, 7

Macaulay E., Wehus I. K., Eriksen H. K., 2013, Phys. Rev. Lett., 111, 161301

Moresco M. et al., 2012, J. Cosmol. Astropart. Phys., 1208, 006

Moresco M., 2015, MNRAS, 450, L16

Moresco M. et al., 2016, J. Cosmol. Astropart. Phys., 1605, 014

Mukhanov V. F., Feldman H. A., Brandenberger R. H., 1992, Phys. Rep., 215,203

Murgia R., Gariazzo S., Fornengo N., 2016, J. Cosmol. Astropart. Phys., 1604, 014

Overduin J. M., Cooperstock F. I., 1998, Phys. Rev. D, 58, 043506

Padmanabhan T., 2003, Phys. Rep., 380, 235

Peccei R. D., Solà J., Wetterich C., 1987, Phys. Lett. B, 195, 183

Peebles P. J. E., 1993, Principles of Physical Cosmology. Princeton Univ. Press, Princeton, NJ

Peebles P. J. E., Ratra B., 1988, ApJ, 325, L17

Peebles P. J. E., Ratra B., 2003, Rev. Mod. Phys., 65, 559

Perico E. L. D., Lima J. A. S., Basilakos S., Solà J., 2013, Phys. Rev. D, 88, 063531

Perlmutter S. et al., 1999, ApJ, 517, 565

Planck Collaboration XVI, 2014, A\&A, 571, A16

Planck Collaboration XIII, 2016, A\&A, 594, A13

Planck Collaboration XIV, 2016, A\&A, 594, A14

Pouri A., Basilakos S., Plionis M., 2014, J. Cosmol. Astropart. Phys., 1408, 042

Ratra B., Peebles P. J. E., 1988, Phys. Rev. D, 37, 3406

Riess A. G. et al., 1998, AJ, 116, 1009

Ross A. J. et al., 2015, MNRAS, 449, 835

Sahni V., Starobinsky A. A., 2000, Int. J. Mod. Phys. A, 9, 373

Salim J. M., Waga I., 1993, Class. Quantum Gravity, 10, 1767

Salvatelli V., Said N., Bruni M., Melchiorri A., Wands D. s., 2014, Phys. Rev. Lett., 113, 181301

Schwarz G., 1978, Ann. Stat., 6, 461

Simon J., Verde L., Jiménez R., 2005, Phys. Rev. D, 71, 123001

Simpson F. et al., 2016, Phys. Rev. D, 93, 023525

Solà J., 2008, J. Phys. A, 41, 164066

Solà J., 2011, J. Phys. Conf. Ser., 283, 012033

Solà J., 2013, J. Phys. Conf. Ser., 453, 012015

Solà J., 2015, Int. J. Mod. Phys. D, 24, 1544027 
Solà J., 2016, Int. J. Mod. Phys. A, 31, 1630035

Solà J., Gómez-Valent A., 2015, Int. J. Mod. Phys. D, 24, 1541003

Solà J., Gómez-Valent A., de Cruz Pérez J., 2015, ApJ, 811, L14

Solà J., Gómez-Valent A., de Cruz Pérez J., 2017a, ApJ, 836, 43

Solà J., Gómez-Valent A., de Cruz Pérez J., 2017b, Mod. Phys. Lett. A, 32, 1750054

Solà J., Gómez-Valent A., de Cruz Pérez J., 2017c, Int. J. Mod. Phys. A, 32, 1730014

Solà J., Gómez-Valent A., de Cruz Pérez J., 2017d, Phys. Lett. B, 774, 317

Solà, de Cruz Pérez J., Gómez-Valent A., 2018, Europhys. Lett., 121, 39001

Song Y.-S., Percival W. J., 2009, J. Cosmol. Astropart. Phys., 0910, 004

Springob C. M. et al., 2016, MNRAS, 456, 1886

Stern D. et al., 2010, J. Cosmol. Astropart. Phys., 1002, 008

Terazawa H., 1981, Phys. Lett. B, 101, 43

Turner S. M., White M., 1997, Phys. Rev. D, 56, R4439

Valentino E. D., Melchiorri A., Linder E. V., Silk J., 2017a, Phys. Rev. D, 96, 023523

Valentino E. D., Melchiorri A., Mena O., 2017b, Phys. Rev. D, 96, 043503

Wang L. M., Steinhardt P. J., 1998, ApJ, 508, 483
Wang Y. et al., 2015, Phys. Rev. D, 92, 103005

Weinberg S., 1989, Rev. Mod. Phys., 61, 1

Weiss N., 1987, Phys. Lett. B, 197, 42

Wetterich C., 1988, Nucl. Phys. B, 302, 668

Wetterich A., 1995, A\&A, 301, 321

WMAP Collaboration, 2013, ApJS, 208, 19

Yu H., Ratra B., Wang F.-Y., 2018, ApJ, 856, 3

Zee A., 1985, Phys. Lett. B, 161, 141

Zeldovich Y. B., 1967, JETP Lett., 6, 316

Zhai Z., Blanton M., Slosar A., Tinker J., 2017, ApJ, 850, 183

Zhang C., Zhang H., Yuan S., Liu S., Zhang T.-J, Sun Y.-C, 2014, Res. Astron. Astrophys., 14, 1221

Zhao G.-B., Li Y.-H., Zhang J.-F., Zhang X., 2017a, Nat. Astron., 1, 627

Zhao M.-M. et al., 2017b, MNRAS, 469, 1713

Zlatev I., Wang L. M., Steinhardt P. J., 1999, Phys. Rev. Lett., 82, 896

This paper has been typeset from a $\mathrm{T}_{\mathrm{E}} \mathrm{X} / \mathrm{L} \mathrm{T} \mathrm{E} \mathrm{X}$ file prepared by the author. 